
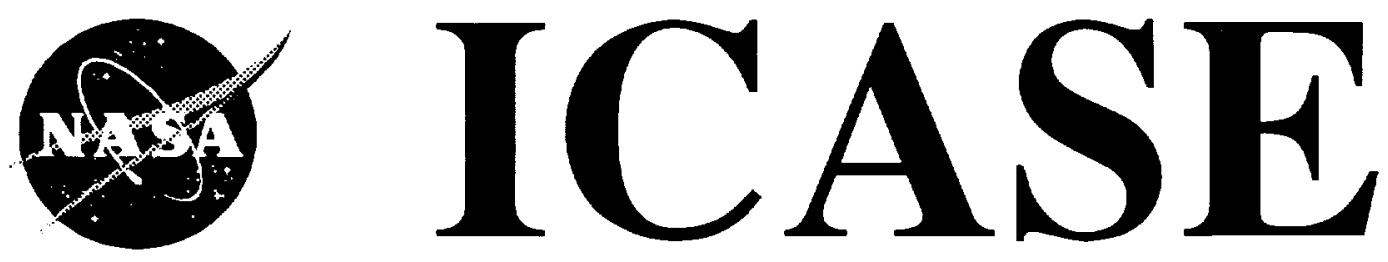

\title{
ACCURATE FINITE DIFFERENCE METHODS FOR TIME-HARMONIC WAVE PROPAGATION
}

Isaac Harari Eli Turkel

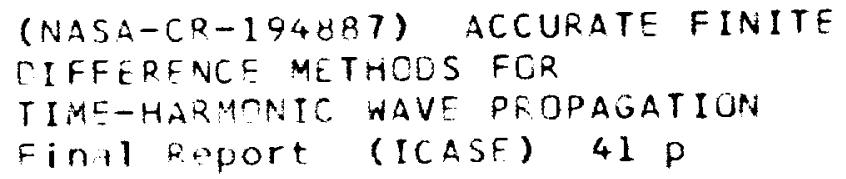

Contract NAS1-19480

March 1994

Institute for Computer Applications in Science and Engineering NASA Langley Research Center

Hampton, VA 23681-(0001 



\title{
Accurate Finite Difference Methods for Time-harmonic Wave Propagation*
}

\author{
Isaac Harari \\ Tel-Aviv University \\ Eli Turkel \\ Tel-Aviv University \\ and \\ Institute for Computer Applications in Science and Engineering
}

\begin{abstract}
Finite difference methods for solving problems of time-harmonic acoustics are developed and analyzed. Multi-dimensional inhomogeneous problems with variable, possibly discontinuous, coefficients are considered, accounting for the effects of employing non-uniform grids. A weighted-average representation is less sensitive to transition in wave resolution (due to variable wave numbers or non-uniform grids) than the standard pointwise representation. Further enhancement in method performance is obtained by basing the stencils on generalizations of Padé approximation, or generalized definitions of the derivative, reducing spurious dispersion, anisotropy and reflection, and by improving the representation of source terms. The resulting schemes have fourth-order accurate local truncation error on uniform grids and third order in the non-uniform case. Guidelines for discretization pertaining to grid orientation and resolution are presented.
\end{abstract}

*This research was supported by the National Aeronautics and Space Administration under NASA Contract No. NAS1-19480 while the authors were in residence at the Institute for Computer Applications in Science and Engineering (ICASE), NASA Langley Research Center, Hampton, VA 23681. The first author was also supported by the Center for Absorption in Science, Ministry of Immigrant Absorption, State of Israel. 



\section{Contents}

Abstract

1 Introduction 1

2 Discrete Formulations 2

2.1 One dimension . . . . . . . . . . . . . . 3

2.2 Two dimensions ................... 6

3 Spurious Dispersion $\quad 9$

4 Spurious Anisotropy $\quad 13$

5 Spurious Reflection and Transmission 21

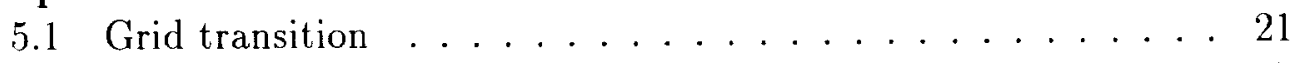

5.2 Interface of physical properties. . . . . . . . . . 27

6 Local Truncation Error Analysis $\quad 30$

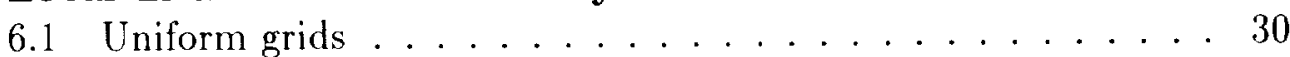

6.2 Non-uniform grids . . . . . . . . . . . . . 31

7 Conclusions $\quad \mathbf{3 4}$

$\begin{array}{lr}\text { Acknowledgement } & \mathbf{3 5}\end{array}$

$\begin{array}{ll}\text { References } & 35\end{array}$

PAGE 11 INTENTIONALLY BLANK, .. 



\section{Introduction}

Boundary-value problems governed by the Helmholtz equation model propagation and evanescence of time-harmonic waves, describing a variety of physical phenomena, including acoustic, elastic and electromagnetic waves. When the wavelength is of the same order as characteristic length scales asymptotic methods usually cannot be employed and standard numerical methods such as boundary element, finite element or finite difference methods are often required.

Finite difference methods are a prevalent computational technique that applies to variable coefficient as well as nonlinear problems. A general framework for deriving higher-order finite difference schemes was proposed by Lynch and Rice for ordinary differential equations [1] and elliptic partial differential equations [2], and applied to the Helmholtz equation [3]. The coefficients of the stencil in this method are computed by solving a local system of equations so that it is exact on a given space of polynomials. This is repeated at every gird point at which the solution is unknown, contributing to the cost of computation. Accuracy is further enhanced by judiciously selecting the points at which source terms are evaluated and computing their coefficients in the same way.

A family of finite difference schemes with improved representation of a range of wave numbers is presented and analyzed in [4]. Tam and Webb [5] optimize the dispersion properties of higher-order finite difference discretization of the linearized Euler equations. In this approach the order of accuracy of a stencil is allowed to drop, freeing a parameter that is then designed to improve dispersion response.

Finite difference equations can be obtained by replacing the limit that defines the derivative with a finite counterpart. The familiar definition of the derivative may be generalized by introducing a resolution-dependent parameter leading to improved performance of the discrete methods. As long as the limit behavior is unaltered consistency of the approximation is retained. This idea was introduced by Mickens and employed as a means of generating stable finite difference schemes on uniform grids for several differential equations in one spatial dimension $([6,7]$ and references therein). Similar discrete equations are obtained by new classes of finite element methods for a variety of applications, including wave propagation (e.g., [8] and references therein). It should be noted that analysis of the computation of waves [9] 
indicates that accuracy depends not only on the product of wave number and grid size (related to resolution), but also on product of other powers of these quantities.

In this work we apply generalizations of the definition of the derivative and of Padé approximation, to finite difference stencils for the Helmholtz equation in order to obtain improved dispersion behavior. Contrary to HODIE methods, the coefficients are obtained explicitly. Multi-dimensional inhomogeneous problems with variable, possibly discontinuous, coefficients are considered, accounting for the effects of employing non-uniform grids. Several finite difference stencils in one and two dimensions are presented in Sec. 2. The analysis of the numerical methods gradually builds up to the general case. Performance of the various formulations for homogeneous problems with constant coefficients on uniform grids is examined by dispersion analysis in Sec. 3. This tool is employed to define the resolution-dependent parameter for improved performance. In Sec. 4 the effect of the direction of wave propagation relative to grid lines is accounted for. The effects of non-uniform grids and discontinuities in physical properties are investigated in Sec. 5. Standard finite difference methods are often not well-suited for interface problems (see, e.g., [10, pp. 17-21]). However, appropriate representation preserves the order of accuracy of the continuous-coefficient, and even constant-coefficient case. (Issues related to curved interfaces, as well as curved domain boundaries are not treated herein.) The results of these analyses are corroborated by means of local truncation error analysis in Sec. 6, accounting also for the effects of source terms. Numerical testing of these stencils will be performed in future work.

\section{Discrete Formulations}

The Helmholtz equation is

$$
\Delta \phi+k^{2} \phi+f=0
$$

where $k=\omega / c_{0}$ is the wave number, $\omega$ is the angular frequency and $c_{0}$ is the speed of sound, and $f$ is a given source distribution. Although not explicitly addressed in the following, the case of $k^{2}<0$ which corresponds to evanescent waves or singular diffusion problems is also covered. An inhomogeneous medium is represented by spatial variations in $k(x)$. 


\subsection{One dimension}

Consider a uniform grid of size $h$ with points at $x_{j}=j h$. A typical starting point is based on the standard finite difference stencil for the second derivative

$$
D_{x x} \phi_{j}:=\frac{\phi_{j+1}-2 \phi_{j}+\phi_{j-1}}{h^{2}}
$$

and pointwise (PT) representation of undifferentiated terms

$$
D_{x x} \phi_{j}+k^{2} \phi_{j}+f_{j}=0
$$

where $\phi_{j}$ is the discrete solution at point $j$ and $f_{j}=f\left(x_{j}\right)$. On a non-uniform grid this generalizes to

$$
\left(\frac{\phi_{j+1}-\phi_{j}}{h^{+}}-\frac{\phi_{j}-\phi_{j-1}}{h^{-}}\right) / \frac{h^{+}+h^{-}}{2}+k^{2} \phi_{j}+f_{j}=0
$$

where $h^{-}$and $h^{+}$are the grid size before and after point $j$, respectively. For a discontinuity in physical properties at point $j$ the stencil becomes

$$
\left(\frac{\phi_{j+1}-\phi_{j}}{h^{+}}-\frac{\phi_{j}-\phi_{j-1}}{h^{-}}\right) / \frac{h^{+}+h^{-}}{2}+\frac{\left(k^{+}\right)^{2} h^{+}+\left(k^{-}\right)^{2} h^{-}}{h^{+}+h^{-}} \phi_{j}+f_{j}=0
$$

where $k^{-}$and $k^{+}$are the wave numbers before and after point $j$, respectively. These may be also considered as piecewise-constant approximations of variable coefficients.

The undifferentiated terms may be represented by a weighted average (WA) suggested by linear finite elements (with piecewise linear approximation of the source distribution, see, e.g., [11, pp. 45-46])

$$
D_{x x} \phi_{j}+k^{2} \frac{\phi_{j+1}+4 \phi_{j}+\phi_{j-1}}{6}+\frac{f_{j+1}+4 f_{j}+f_{j-1}}{6}=0
$$

This scheme has the same asymptotic behavior as the pointwise representation, but improvement in performance on coarse grids is evident (see Sec. 3). For variable coefficients this becomes

$$
D_{x x} \phi_{j}+\frac{\left(k^{2} \phi\right)_{j+1}+4\left(k^{2} \phi\right)_{j}+\left(k^{2} \phi\right)_{j-1}}{6}+\frac{f_{j+1}+4 f_{j}+f_{j-1}}{6}=0
$$

where $\left(k^{2} \phi\right)_{j}=k^{2}\left(x_{j}\right) \phi_{j}$. 
On a non-uniform grid the appropriate weighting is

$$
\begin{gathered}
\left(\frac{\phi_{j+1}-\phi_{j}}{h^{+}}-\frac{\phi_{j}-\phi_{j-1}}{h^{-}}\right) / \frac{h^{+}+h^{-}}{2}+ \\
\frac{k^{2}}{3}\left(\frac{h^{+}}{h^{+}+h^{-}}\left(\phi_{j+1}+2 \phi_{j}\right)+\frac{h^{-}}{h^{+}+h^{-}}\left(2 \phi_{j}+\phi_{j-1}\right)\right)+ \\
\frac{1}{3}\left(\frac{h^{+}}{h^{+}+h^{-}}\left(f_{j+1}+2 f_{j}\right)+\frac{h^{-}}{h^{+}+h^{-}}\left(2 f_{j}+f_{j-1}\right)\right)=0
\end{gathered}
$$

Superior performance on non-uniform grids (see Sec. 5) is attained with no increase in the number of points in the stencil. For a discontinuity in physical properties at point $j$ the stencil becomes

$$
\begin{gathered}
\left(\frac{\phi_{j+1}-\phi_{j}}{h^{+}}-\frac{\phi_{j}-\phi_{j-1}}{h^{-}}\right) / \frac{h^{+}+h^{-}}{2}+ \\
\frac{1}{3}\left(\frac{\left(k^{+}\right)^{2} h^{+}}{h^{+}+h^{-}}\left(\phi_{j+1}+2 \phi_{j}\right)+\frac{\left(k^{-}\right)^{2} h^{-}}{h^{+}+h^{-}}\left(2 \phi_{j}+\phi_{j-1}\right)\right)+ \\
\frac{1}{3}\left(\frac{h^{+}}{h^{+}+h^{-}}\left(f_{j+1}+2 f_{j}\right)+\frac{h^{-}}{h^{+}+h^{-}}\left(2 f_{j}+f_{j-1}\right)\right)=0
\end{gathered}
$$

Again, this may also be considered as a piecewise-constant approximation of the case of variable coefficients.

Performance of finite difference schemes for the Helmholtz equation may be enhanced by basing the stencils on more general definitions of the derivative

$$
\frac{d \phi}{d x}=\lim _{h \rightarrow 0} \frac{\phi(x+h)-\phi(x)}{\beta(k h) h}
$$

where, for consistency

$$
\lim _{k h \rightarrow 0} \beta=1
$$

This definition depends on $k h$, an indication of wave resolution by the grid. For the Laplacian this reduces to the standard definition for grids of any size.

This generalization of the derivative definition may be applied for either the first or second derivatives, or to both. On uniform grids all are equivalent. Since the parameter depends on the grid size it is applicable to non-uniform grids as well. Superior performance on non-uniform grids is obtained by 
applying this concept to the second derivative alone (see Sec. 5). For the uniform case this reduces to

$$
\frac{\phi_{j+1}-2 \phi_{j}+\phi_{j-1}}{\beta h^{2}}+k^{2} \frac{\phi_{j+1}+4 \phi_{j}+\phi_{j-1}}{6}+\frac{f_{j+1}+4 f_{j}+f_{j-1}}{6}=0
$$

The resolution-dependent parameter $\beta$ is defined to improve method performance. For example, the parameter may be defined to eliminate numerical dispersion

$$
\beta=\frac{6}{(k h)^{2}} \frac{1-\cos (k h)}{2+\cos (k h)}
$$

so that in simplified settings the phase is exact (EP), resulting in no truncation error under some circumstances. This definition satisfies the consistency requirement (11). In such cases the representation of source terms, which is exact for piecewise linear variation, is no longer sufficiently accurate. A modification of the representation of source terms that does not degrade the higher-order accuracy of such schemes, similar to that employed by HODIE methods [2], is

$$
\frac{\phi_{j+1}-2 \phi_{j}+\phi_{j-1}}{\beta h^{2}}+k^{2} \frac{\phi_{j+1}+4 \phi_{j}+\phi_{j-1}}{6}+\frac{f_{j+1 / 2}+f_{j}+f_{j-1 / 2}}{3}=0
$$

(suggested by linear finite elements with piecewise quadratic approximation of the source distribution) where $f_{j+1 / 2}$ is the load term evaluated at the midpoint. For a piecewise linear source distribution this is identical to (12).

One possibility of the parameter

$$
\beta=\frac{12}{12-(k h)^{2}}
$$

yields high-order representation

$$
D_{x x} \phi_{j}+k^{2} \frac{\phi_{j+1}+10 \phi_{j}+\phi_{j-1}}{12}+\frac{f_{j+1 / 2}+f_{j}+f_{j-1 / 2}}{3}=0
$$

(Ho). This stencil (without the modification in the representation of source terms) may also be derived by employing Padé approximation

$$
\frac{D_{x x}}{1+h^{2} / 12 D_{x x}} \phi_{j}+k^{2} \phi_{j}+f_{j}=0
$$


(see, e.g., [12, p. 538]).

This concept, in its original form, which may be viewed as an average of the pointwise and weighted-average representations of the undifferentiated term [13], provides high-order performance on uniform grids, but severely degrades in the non-uniform case. However, an appropriate generalization to non-uniform grids, based on the concept of generalizing the derivative definition, leads to improved performance in the general case as well (see Secs. 5 and 6). Allowing discontinuities in physical coefficients and accounting for non-uniform grids the proposed scheme is

$$
\begin{gathered}
\left(\frac{\phi_{j+1}-\phi_{j}}{h^{+}}-\frac{\phi_{j}-\phi_{j-1}}{h^{-}}\right) / \frac{\beta^{+} h^{+}+\beta^{-} h^{-}}{2}+ \\
\frac{1}{3}\left(\frac{\left(k^{+}\right)^{2} \beta^{+} h^{+}}{\beta^{+} h^{+}+\beta^{-h^{-}}}\left(\phi_{j+1}+2 \phi_{j}\right)+\frac{\left(k^{-}\right)^{2} \beta^{-} h^{-}}{\beta^{+} h^{+}+\beta^{-} h^{-}}\left(2 \phi_{j}+\phi_{j-1}\right)\right)+ \\
\frac{1}{3}\left(\frac{\beta^{+} h^{+}}{\beta^{+} h^{+}+\beta^{-} h^{-}}\left(2 f_{j+1 / 2}+f_{j}\right)+\frac{\beta^{-} h^{-}}{\beta^{+} h^{+}+\beta^{-} h^{-}}\left(f_{j}+2 f_{j-1 / 2}\right)\right)=0
\end{gathered}
$$

where $\beta^{ \pm}=\beta\left(k^{ \pm} h^{ \pm}\right)$.

\subsection{Two dimensions}

Consider a two-dimensional uniform grid of size $h$ with points at $x_{i}=i h$ and $y_{j}=j h$. For simplicity we consider the homogeneous case. A typical starting point is the five-point representation

$$
\begin{aligned}
D_{x x} \phi_{i, j}+D_{y y} \phi_{i, j}+k^{2} \phi_{i, j} & = \\
\frac{\phi_{i+1, j}-2 \phi_{i, j}+\phi_{i-1, j}}{h^{2}}+\frac{\phi_{i, j+1}-2 \phi_{i, j}+\phi_{i, j-1}}{h^{2}}+k^{2} \phi_{i, j} & =0
\end{aligned}
$$

which is the two-dimensional analog of (3). Non-uniform grids and material discontinuities may be accounted for by generalizations of (4) and (5).

The two-dimensional counterpart of the idea that leads to (6), obtained by employing bilinear finite elements, is a nine-point representation

$$
\begin{aligned}
& \frac{\phi_{i+1, j-1}+2 \phi_{i+1, j}+\phi_{i+1, j+1}-8 \phi_{i, j}+\phi_{i-1, j-1}+2 \phi_{i-1, j}+\phi_{i-1, j+1}}{h^{2}}+ \\
& \frac{\phi_{i-1, j+1}+2 \phi_{i, j+1}+\phi_{i+1, j+1}-8 \phi_{i, j}+\phi_{i-1, j-1}+2 \phi_{i, j-1}+\phi_{i+1, j-1}}{h^{2}}+
\end{aligned}
$$




$$
\begin{aligned}
\frac{k^{2}}{6}\left(\phi_{i+1, j+1}+4 \phi_{i+1, j}+4 \phi_{i, j+1}+\phi_{i+1, j-1}+16 \phi_{i, j}+\right. & (20) \\
\left.\phi_{i-1, j+1}+4 \phi_{i, j-1}+4 \phi_{i-1, j}+\phi_{i-1, j-1}\right) & =0
\end{aligned}
$$

leading to a significant reduction in spurious anisotropy (see Sec. 4). HODIE methods [2] also employ nine-point stencils in two dimensions. The bandwidth of the ensuing linear algebra problem is typically slightly larger but the difference in the cost of computation is insignificant.

Performance can again be improved by substituting $\beta h$ for $h$ as in the onedimensional case (14), based on the same definitions (13) and (15), although the methods are higher order only for propagation along grid lines.

In order to maintain higher-order performance on uniform grids in two dimensions in all directions of propagation, the Padé approximation concept is employed. The two-dimensional counterpart of (17) is

$$
\frac{D_{x x}}{1+\frac{h^{2}}{12} D_{x x}} \phi_{i, j}+\frac{D_{y y}}{1+\frac{h^{2}}{12} D_{y y}} \phi_{i, j}+k^{2} \phi_{i, j}=0
$$

This may be generalized to

$$
\begin{aligned}
& \left(1+\frac{h^{2}}{12} D_{y y}\right) D_{x x} \phi_{i, j}+\left(1+\frac{h^{2}}{12} D_{x x}\right) D_{y y} \phi_{i, j}+ \\
& k^{2}\left(1+\frac{h^{2}}{12}\left(D_{x x}+D_{y y}\right)+\gamma \frac{h^{4}}{144} D_{x x} D_{y y}\right) \phi_{i, j}=0
\end{aligned}
$$

where $\gamma$ is selected to further improve properties in directions other than along grid lines, without effecting dispersion along grid lines and without degrading higher-order behavior in all directions.

The standard Padé approximation is obtained by selecting $\gamma=1$ which yields the scheme

$$
\begin{gathered}
\left(D_{x x}+D_{y y}+\frac{h^{2}}{6} D_{x x} D_{y y}\right) \phi_{i, j}+ \\
\frac{k^{2}}{12}\left(\phi_{i+1, j+1}+10 \phi_{i+1, j}+10 \phi_{i, j+1}+\phi_{i+1, j-1}+100 \phi_{i, j}+\right. \\
\left.\phi_{i-1, j+1}+10 \phi_{i, j-1}+10 \phi_{i-1, j}+\phi_{i-1, j-1}\right)=0
\end{gathered}
$$


where

$$
\begin{array}{r}
\left(D_{x x}+D_{y y}+\frac{h^{2}}{6} D_{x x} D_{y y}\right) \phi_{i, j}= \\
\frac{\phi_{i+1, j-1}+8 \phi_{i+1, j}+\phi_{i+1, j+1}-20 \phi_{i, j}+\phi_{i-1, j-1}+8 \phi_{i-1, j}+\phi_{i-1, j+1}}{h^{2}}+ \\
\frac{\phi_{i-1, j+1}+8 \phi_{i, j+1}+\phi_{i+1, j+1}-20 \phi_{i, j}+\phi_{i-1, j-1}+8 \phi_{i, j-1}+\phi_{i+1, j-1}}{h^{2}}
\end{array}
$$

Neglecting higher-order terms in the Padé approximation by selecting $\gamma=0$ leads to the slightly simplified stencil presented in $[12$, p. 542]

$$
\begin{gathered}
\left(D_{x x}+D_{y y}+\frac{h^{2}}{6} D_{x x} D_{y y}\right) \phi_{i, j}+ \\
k^{2}\left(\phi_{i+1, j}+\phi_{i, j+1}+8 \phi_{i, j}+\phi_{i, j-1}+\phi_{i-1, j}\right)=0
\end{gathered}
$$

The computational cost is essentially unaffected since the bandwidth of the algebraic equations is identical. Another alternative presented in [12, p. 542] is

$$
\begin{gathered}
\left(D_{x x}+D_{y y}+\frac{h^{2}}{6} D_{x x} D_{y y}\right) \phi_{i, j}+ \\
\frac{k^{2}}{6}\left(\phi_{i+1, j+1}+4 \phi_{i+1, j}+4 \phi_{i, j+1}+\phi_{i+1, j-1}+52 \phi_{i, j}+\right. \\
\left.\phi_{i-1, j+1}+4 \phi_{i, j-1}+4 \phi_{i-1, j}+\phi_{i-1, j-1}\right)=0
\end{gathered}
$$

obtained by selecting $\gamma=2$.

Other values for $\gamma$ lead to other alternatives. In Sec. 4 it is seen that selecting $\gamma=2 / 5$, which leads to the stencil

$$
\begin{gathered}
\left(D_{x x}+D_{y y}+\frac{h^{2}}{6} D_{x x} D_{y y}\right) \phi_{i, j}+ \\
\frac{k^{2}}{30}\left(\phi_{i+1, j+1}+28 \phi_{i+1, j}+28 \phi_{i, j+1}+\phi_{i+1, j-1}+244 \phi_{i, j}+\right. \\
\left.\phi_{i-1, j+1}+28 \phi_{i, j-1}+28 \phi_{i-1, j}+\phi_{i-1, j-1}\right)=0
\end{gathered}
$$

minimizes dispersion along the diagonals. On the other hand, reducing sensitivity of the scheme to direction of propagation is attained by the choice of 
$\gamma=14 / 5$, which yields the stencil

$$
\begin{gathered}
\left(D_{x x}+D_{y y}+\frac{h^{2}}{6} D_{x x} D_{y y}\right) \phi_{i, j}+ \\
\frac{k^{2}}{30}\left(7 \phi_{i+1, j+1}+16 \phi_{i+1, j}+16 \phi_{i, j+1}+7 \phi_{i+1, j-1}+268 \phi_{i, j}+\right. \\
\left.7 \phi_{i-1, j+1}+16 \phi_{i, j-1}+16 \phi_{i-1, j}+7 \phi_{i-1, j-1}\right)=0
\end{gathered}
$$

All these alternatives reduce to (HO) in one dimension. Thus the dispersion analysis for (HO) in Sec. 3 describes the dispersion of all alternatives along grid lines. In Sec. 4 the performance of various alternatives in other directions is compared.

\section{Spurious Dispersion}

A homogeneous, isotropic continuum is non-dispersive. Waves travel at a phase velocity

$$
c_{p}:=\frac{\omega}{k}=c_{0}
$$

and energy propagates at the group velocity

$$
c_{g}:=\frac{\partial \omega}{\partial k}=c_{0}
$$

and so both are identical.

For the discrete representation this is usually no longer the case. Each stencil can be characterized by an approximate wave number $k^{h}=k^{h}(k h)$, which depends on wave resolution and thus accounts for numerical dispersion. The phase velocity in the discrete case is

$$
c_{p}^{h}=\frac{\omega}{k^{h}}=\frac{k}{k^{h}} c_{0}
$$

and the numerical group velocity is

$$
\begin{aligned}
c_{g}^{h} & =\frac{\partial \omega}{\partial k^{h}} \\
& =\frac{\partial \omega}{\partial k} \frac{\partial k}{\partial k^{h}} \\
& =\left(\frac{\partial k^{h}}{\partial k}\right)^{-1} c_{0}
\end{aligned}
$$


On a uniform grid in one dimension a numerical solution in the form of a plane wave is

$$
\phi_{j}=\exp \left(i k^{h} h\right)^{j}
$$

PT For point $j$ the pointwise representation (3) of the plane wave solution yields

$$
\begin{aligned}
0 & =\exp \left(i k^{h} h\right)-\left(2-(k h)^{2}\right)+1 / \exp \left(i k^{h} h\right) \\
& =2 \cos \left(k^{h} h\right)-\left(2-(k h)^{2}\right)
\end{aligned}
$$

leading to the dispersion relation

$$
k^{h} h=\arccos \left(1-(k h)^{2} / 2\right)
$$

In one dimension the number of grid points in a wave is

$$
G=2 \pi /(k h)
$$

The discrete solution represents propagation in the range $k h<2$ which corresponds to a limit of approximately three grid points per wavelength. Within this range the numerical phase velocity is thus

$$
c_{p}^{h} / c_{0}=k h / \arccos \left(1-(k h)^{2} / 2\right)
$$

and the numerical group velocity is

$$
c_{g}^{h} / c_{0}=\sqrt{1-(k h)^{2} / 4}
$$

Both are slower than the speed of sound in the material $c_{0}$.

WA Similarly, for the weighted-average representation (6) the dispersion relation is

$$
k^{h} h=\arccos \left(\frac{1-(k h)^{2} / 3}{1+(k h)^{2} / 6}\right)
$$

representing propagation in the range $k h<\sqrt{12}$, a limit of approximately two grid points per wavelength. Within this range the numerical phase velocity is obtained directly from the dispersion relation and the numerical group velocity is

$$
c_{g}^{h} / c_{0}=\sqrt{1-(k h)^{2} / 12}\left(1+(k h)^{2} / 6\right)
$$

Both are faster than the speed of sound in the material. 
EP The resolution-dependent parameter $\beta$ may be defined so that discrete representations are non-dispersive (13) as is the case for the continuum. In one dimension this formulation has zero local truncation error for the homogeneous, constant coefficient case on uniform grids, and the phase and group velocities are exact. Careful generalization leads to improved performance on general configurations.

HO The higher-order representation (15) is an approximation of the exact phase definition (13) on uniform grids. The resulting higher-order dispersion relation

$$
k^{h} h=\arccos \left(\frac{1-5(k h)^{2} / 12}{1+(k h)^{2} / 12}\right)
$$

is a $(1,1)$ Padé approximation, representing propagation in the range $k h<\sqrt{6}$, a limit of approximately $21 / 2$ grid points per wavelength. Within this range the numerical phase velocity is again obtained directly from the dispersion relation and the numerical group velocity is

$$
c_{g}^{h} / c_{0}=\sqrt{1-(k h)^{2} / 6}\left(1+(k h)^{2} / 12\right)
$$

Both are slower than the speed of sound in the material. The power series expansion of the dispersion relation

$$
k^{h} h \approx k h+\frac{(k h)^{5}}{480}+\frac{(k h)^{7}}{12096} \geq k h
$$

demonstrates the higher-order nature of this representation.

Dispersion of the various formulations is plotted in Fig. 1. Note that the region of primary interest is $G>4$, a resolution of at least four grid points per wavelength. Within this region the errors in the pointwise and weightedaverage representations are similar, and the asymptotic behavior is the same (see Sec. 6). However, even approaching the limit of resolution, and certainly beyond it, the weighted-average performance is superior. For example, at the limit of resolution $(G=4)$ there is a $38 \%$ error in the group velocity for the pointwise representation, whereas in the weighted-average representation the error is only $26 \%$. The higher-order method offers significantly superior representation, an error of only $7 \%$ in group velocity at $G=4$, and the exact-phase method provides dispersion-free solutions. 

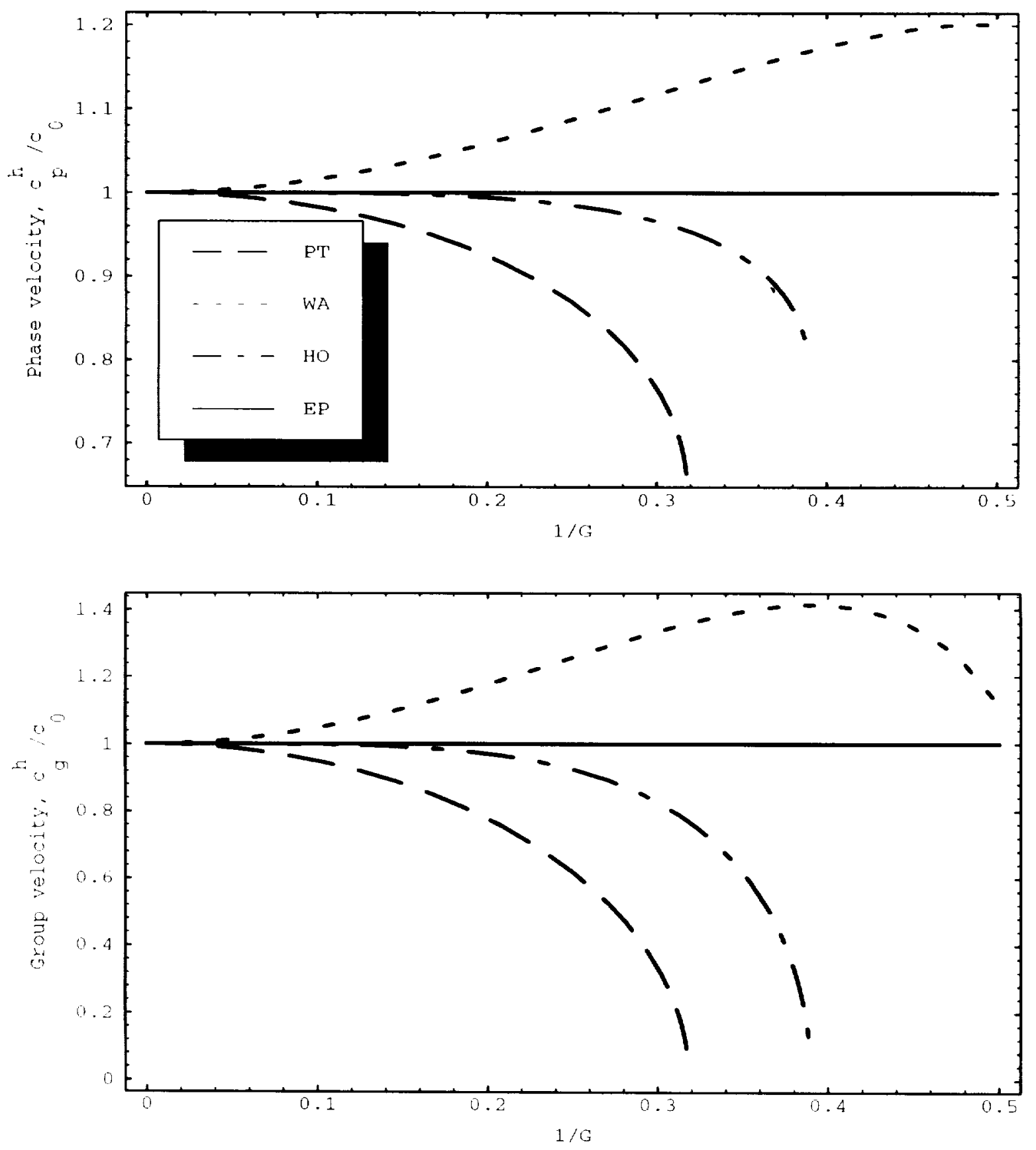

Figure 1: Phase and group velocities of one-dimensionai discrete formulations. 


\section{Spurious Anisotropy}

On the uniform grid in two dimensions a numerical solution in the form of a plane wave oriented at angle $\theta$ to the grid lines is

$$
\phi_{i, j}=\exp \left(i k^{h} h \cos \theta\right)^{i} \exp \left(i k^{h} h \sin \theta\right)^{j}
$$

PT For point $i, j$ the pointwise representation (19) of the plane wave solution yields

$$
\begin{aligned}
& 0= \exp \left(i k^{h} h \sin \theta\right)+\exp \left(i k^{h} h \cos \theta\right)-\left(4-(k h)^{2}\right)+ \\
& 1 / \exp \left(i k^{h} h \cos \theta\right)+1 / \exp \left(i k^{h} h \sin \theta\right) \\
&=2\left(\cos \left(k^{h} h \cos \theta\right)+\cos \left(k^{h} h \sin \theta\right)\right)-\left(4-(k h)^{2}\right)
\end{aligned}
$$

leading to various dispersion relations, depending on the angle of orientation $\theta$. When the wave is aligned with the grid (e.g., $\theta=0$ ) this leads to the one-dimensional dispersion relation (35). The other extreme case occurs when the wave is oriented in the direction of cell diagonals (e.g., $\theta=\pi / 4)$

$$
k^{h} h=\sqrt{2} \arccos \left(1-(k h)^{2} / 4\right)
$$

The discrete solution represents propagation in the range $k h<\sqrt{8}$. Within this range the numerical phase velocity is again obtained directly from the dispersion relation and the numerical group velocity is

$$
c_{g}^{h} / c_{0}=\sqrt{1-(k h)^{2} / 8}
$$

Both are slower than the speed of sound in the material. It is interesting to note that the pointwise representation is more dispersive when waves are oriented along the grid.

WA Similarly, for the weighted-average representation (21) dispersion relations are obtained from

$$
\begin{array}{r}
4\left(6-(k h)^{2}\right)-\left(12+(k h)^{2}\right) \cos \left(k^{h} h \cos \theta\right) \cos \left(k^{h} h \sin \theta\right)- \\
2\left(3+(k h)^{2}\right)\left(\cos \left(k^{h} h \cos \theta\right)+\cos \left(k^{h} h \sin \theta\right)\right)=0
\end{array}
$$


When the wave is aligned with the grid this leads to the known onedimensional dispersion relation (39) and for waves parallel to cell diagonals

$$
k^{h} h=\sqrt{2} \arccos \left(\frac{1-(k h)^{2} / 6}{1+(k h)^{2} / 12}\right)
$$

representing propagation in the range $k h<\sqrt{24}$. Within this range the numerical phase velocity is obtained directly from the dispersion relation and the numerical group velocity is

$$
c_{g}^{h} / c_{0}=\sqrt{1-(k h)^{2} / 24}\left(1+(k h)^{2} / 12\right)
$$

Both are faster than the speed of sound in the material. The weightedaverage representation is also more dispersive for waves that are oriented with the grid.

HO Dispersion relations for the higher-order representation with $\beta$ defined in (15) at various angles of orientation may be found in similar fashion. For waves aligned with cell diagonals the relation is identical to that of the pointwise representation at this angle (46), and the same holds for the wave velocities. This representation is thus higher order only for waves oriented along grid lines.

Representations that are truly higher order in all directions of propagation are based on (22). For waves aligned with the grid this leads to the higher-order dispersion relation (41) and along cell diagonals the relation is

$$
k^{h} h=\sqrt{2} \arccos \left(\frac{6 \sqrt{1+(1-\gamma)(k h)^{4} / 144}-\left(4+(6-\gamma)(k h)^{2} / 12\right)}{2+\gamma(k h)^{2} / 12}\right)
$$

By examining the power series expansion of this relation

$$
k^{h} h \approx k h+(5 \gamma-2) \frac{(k h)^{5}}{5760}+\frac{(k h)^{7}}{96768}
$$

it is clear that the value of $\gamma=2 / 5$ minimizes dispersion in the direction of cell diagonals. On the other hand, $\gamma=14 / 5$ minimizes the difference between the dispersion along grid lines and in the direction of cell 
diagonals, as seen by comparison to the power series expansion of the higher-order dispersion relation along grid lines (43). Dispersion in the direction of cell diagonals for various values of $\gamma$ is plotted in Fig. 2. As expected, the stencil with $\gamma=2 / 5$ is essentially non-dispersive in the region of primary interest with a resolution of at least four grid points per wavelength.

The ratio between numerical dispersion along grid lines and along cell diagonals is shown in Fig. 3. By design, the stencil with $\gamma=14 / 5$ is the least anisotropic in the range of at least four grid points per wavelength. This is corroborated by the polar plots in Fig. 4, showing the variation in phase velocity with angle of orientation for various resolutions. Note that the figure shows $\left(c_{p}^{h} / c_{0}\right)^{4}$ to accentuate deviations from the exact value of unity. As expected, all the schemes perform identically along grid lines, but behavior in other directions is determined by the choice of $\gamma$. Differences among the various cases become more pronounced with reduced resolution, but in general are not extreme. The two schemes that stand out are indeed $\gamma=2 / 5$ which minimizes dispersion along diagonals, and hence overall, and $\gamma=14 / 5$ which reduces anisotropy.

EP The case that is non-dispersive in one dimension (13) may be treated similarly. In this case there is no dispersion for waves aligned with the grid and the dispersion relation for waves in the direction of cell diagonals is

$$
k^{h} h=\sqrt{2} \arccos \left(2 \frac{1+2 \cos (k h)}{5+\cos (k h)}\right)
$$

The numerical phase velocity is again obtained directly from the dispersion relation and the numerical group velocity is

$$
\frac{c_{g}^{h}}{c_{0}}=\sqrt{\frac{7+5 \cos (k h)}{1+\cos (k h)}}\left(\frac{5+\cos (k h)}{\sqrt{216}}\right)
$$

This representation is obviously less dispersive for waves that are oriented with the grid.

Dispersion in the direction of cell diagonals of the various formulations is plotted in Fig. 5. Recall that the region of primary interest is $G>4$, a resolution of at least four grid points per wavelength. Dispersion properties of 

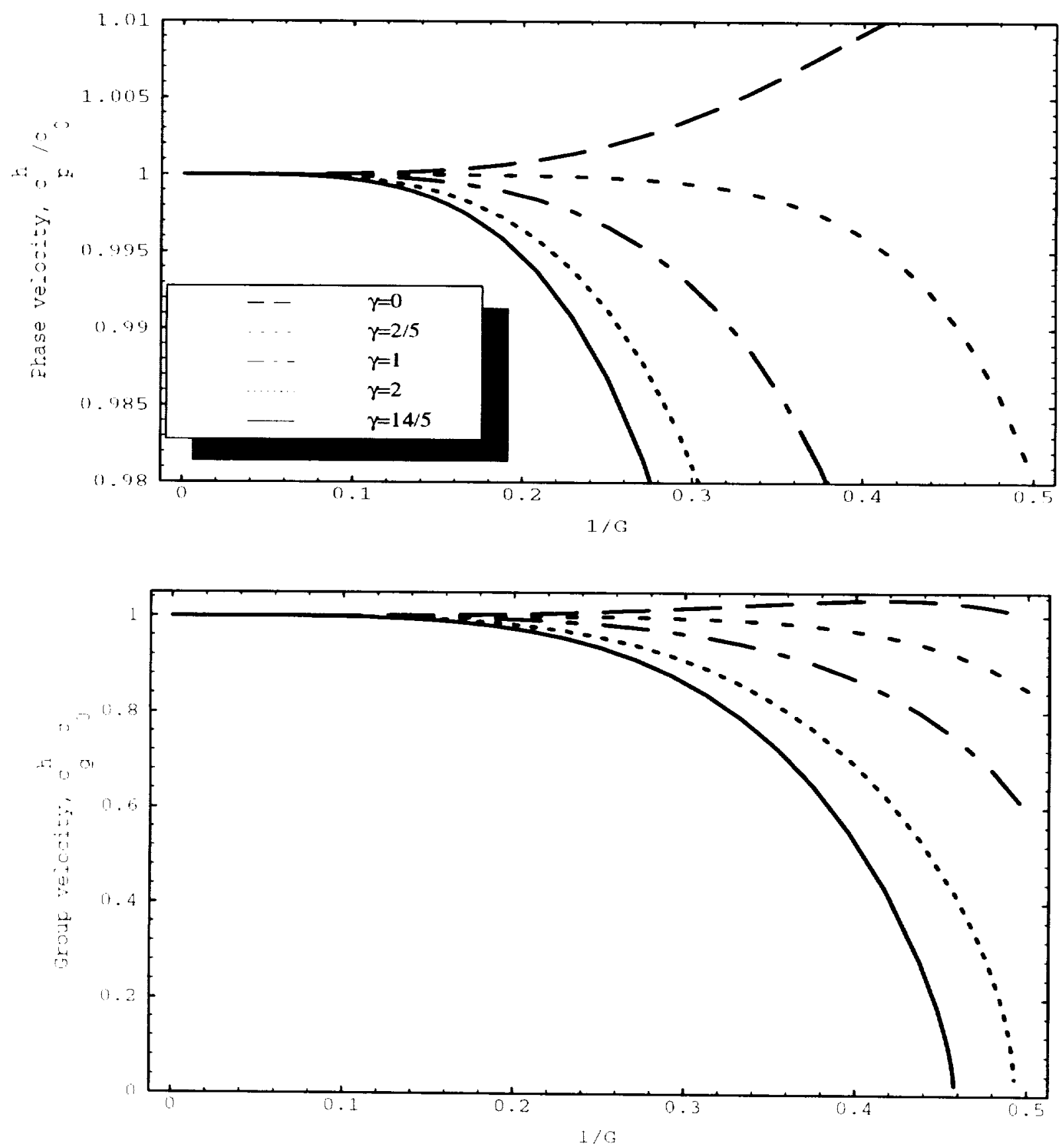

Figure 2: Phase and group velocities along cell diagonals of higher-order discrete formulations based on generalized Padé approximation. 

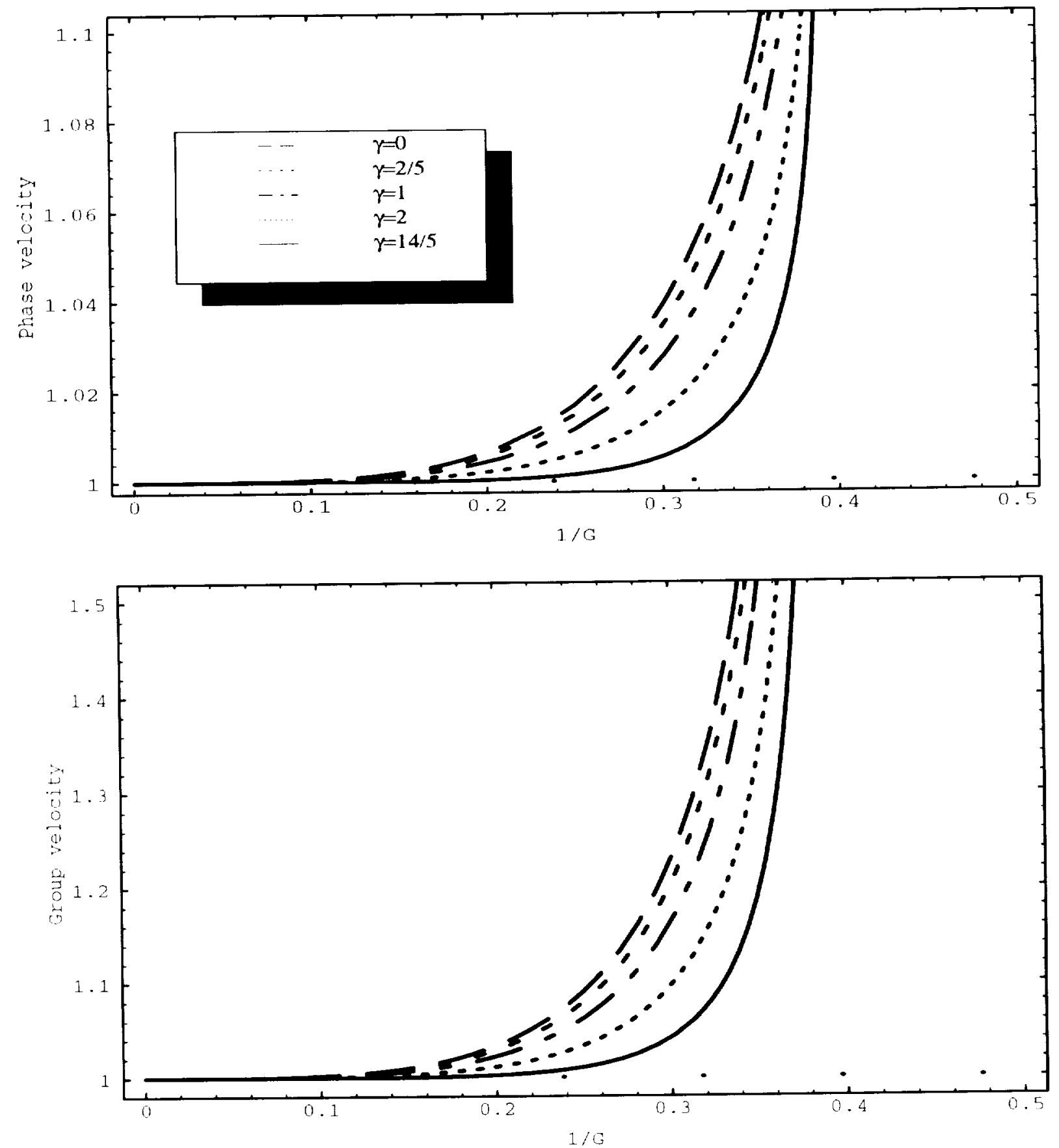

Figure 3: The ratio of numerical dispersion along grid lines and along cell diagonals for higher-order discrete formulations based on generalized Padé approximation. 

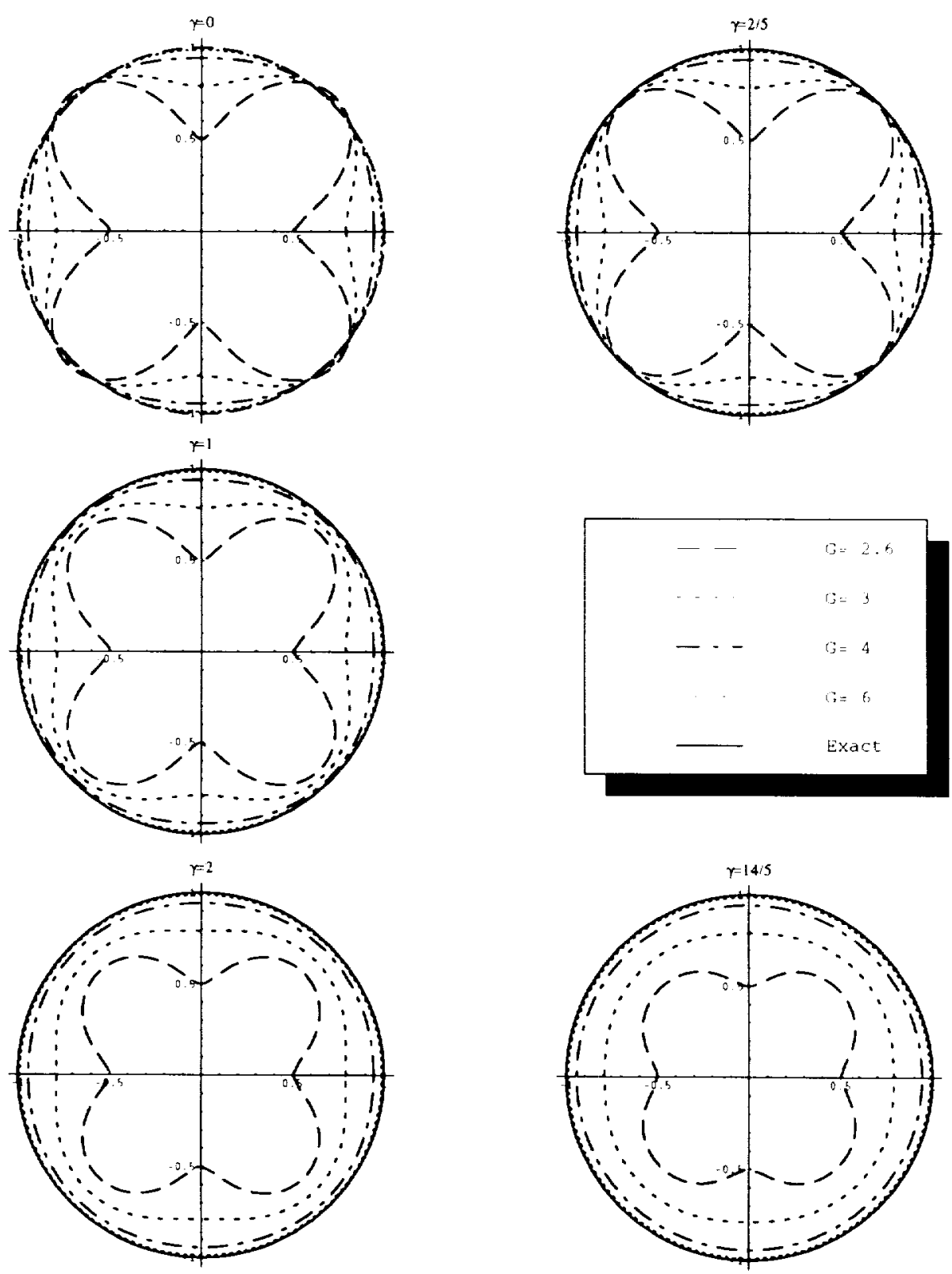

Figure 4: Polar plots of anisotropy in $\left(c_{p}^{h} / c_{0}\right)^{4}$ of higher-order discrete formulations based on generalized Padé approximation. 
each scheme at arbitrary orientations are bounded on one hand by dispersion along grid lines shown in Fig. 1, and on the other hand by dispersion along cell diagonals shown in Fig. 5. Performance of the pointwise and weightedaverage schemes improves the farther the orientation of propagation is from the direction of grid lines. The same holds for the higher-order schemes of interest with $\gamma=2 / 5$ and $\gamma=14 / 5$. For the (EP) method the opposite occurs, so that performance of this scheme is vastly superior along grid lines. This scheme is higher order only along grid lines, but it still maintains a high degree of phase accuracy in all orientations.

The resolution-dependent parameter $\beta$ may be defined so that the numerical representation is non-dispersive for waves at any given angle of orientation. For example

$$
\beta=\frac{12}{(k h)^{2}} \frac{1-\cos (\sqrt{2} k h / 2)}{2+\cos (\sqrt{2} k h / 2)}
$$

eliminates dispersion of waves along cell diagonals. Similar performance was attained in the context of finite element methods [14]. In general, however, the direction of wave propagation is not known in advance and there is a concern that defining $\beta$ for any orientation other than along grid lines may degrade performance on non-uniform grids, as discussed in the following section. Furthermore, grids should be aligned with dominant directions of propagation to the extent possible. For these reasons it is preferred to maintain dispersion-free discrete solutions along grid lines.

Numerical dispersion is thus sensitive to the orientation of wave propagation. The two extreme cases are along grid lines shown in Fig. 1, and along cell diagonals shown in Fig. 5. The largest change in dispersion properties possible is thus the ratio between the two, shown in Fig. 6 . Recall that the region of primary interest is $G>4$, a resolution of at least four grid points per wavelength. For highly resolved phenomena the performance of all schemes is similar and quite good. As wave resolution is reduced only the higherorder schemes (with values of $\gamma$ shown) retain a low level of anisotropy. Of the other schemes, approaching the limit of resolution and certainly beyond it, the pointwise method is clearly more sensitive to direction of propagation. For example, at the limit of resolution $(G=4)$, there is $8 \%$ anisotropy in the pointwise representation of phase velocity, whereas the anisotropy of other methods is at most about half that value. This becomes even more 

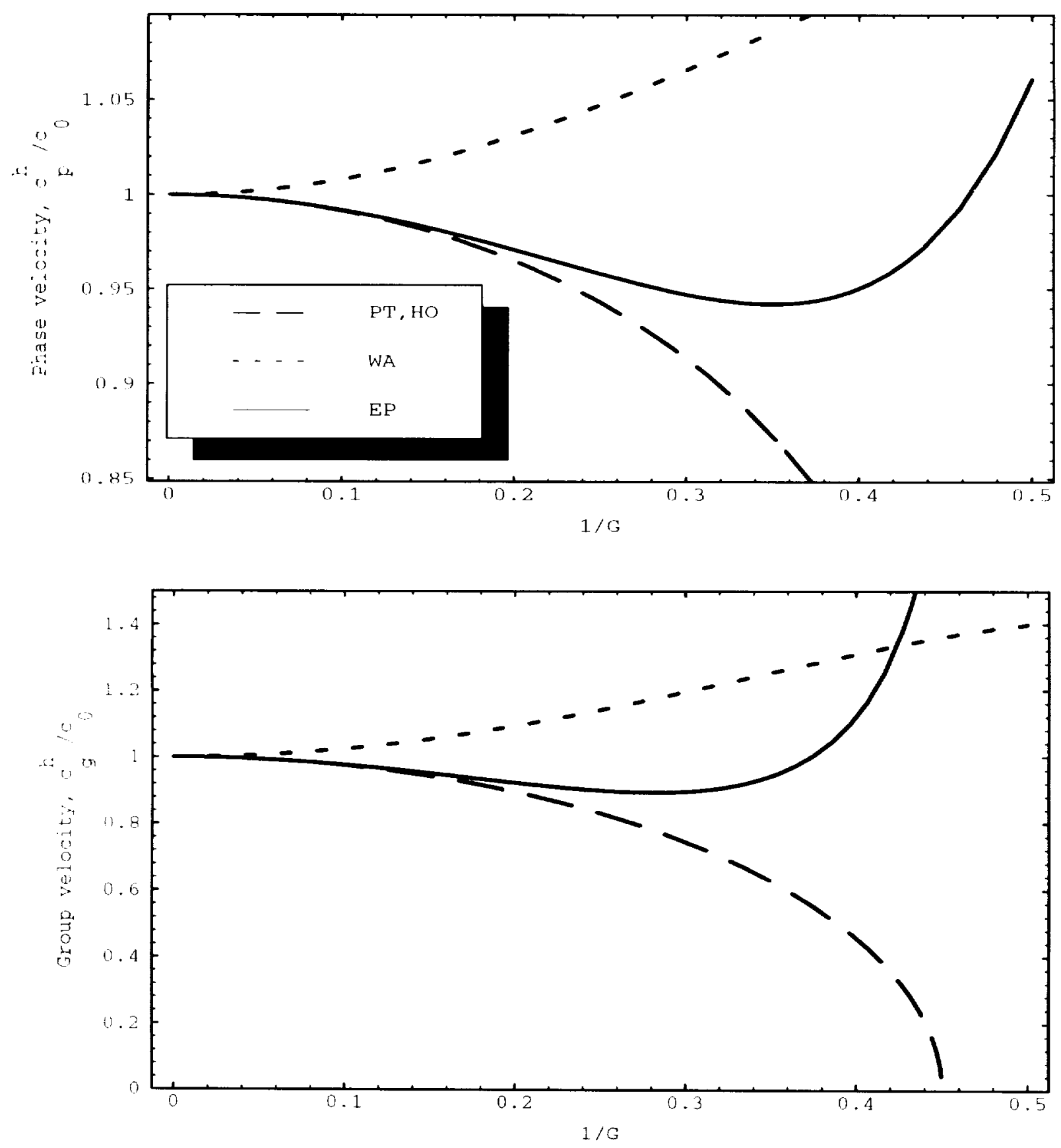

Figure 5: Phase and group velocities of two-dimensional discrete formulations along cell diagonals. 
pronounced in group velocity.

Figure 7 shows the variation in phase velocity with angle of orientation of different schemes at various resolutions. For presentation purposes the figure shows $\left(c_{p}^{h} / c_{0}\right)^{4}$. Note that the plot for (PT) does not include the case of $G=3$ since this scheme no longer represents propagation at this low resolution, which, in any event, is outside the region of primary interest of $G>4$. It is clear from these plots that the numerical phase velocity is less than the speed of sound in the material in all cases shown except for (WA). Close examination of Fig. 4 indicates that this is true of higher-order methods only with $\gamma \geq 2 / 5$. With the exception of (EP), all the schemes considered exhibit superior dispersion behavior along cell diagonals. This would not hold for higher-order methods with $\gamma>14 / 5$, but there is no apparent motivation to pursue such methods in the first place. As mentioned, employing (55) eliminates dispersion along cell diagonals, leading to a version of (EP) with superior dispersion behavior along diagonals that is similar to other schemes in this regard.

Overall, high wave resolution or higher-order methods are required if anisotropy is a concern. Of the methods that are not high order, on grids with lower resolution, the weighted average representation and its enhancements are much less anisotropic than the standard pointwise representation.

\section{Spurious Reflection and Transmission}

Reflected and transmitted waves are generated by incident waves at discontinuities in physical properties. Numerical dispersion of discrete formulations gives rise to incorrect representation of these phenomena at transitions in wave resolution.

\subsection{Grid transition}

In a homogeneous material no reflection should occur. However, changes in grid size alter wave resolution giving rise to spurious reflection and transmission due to numerical dispersion, phenomena that may be characterized in a manner similar to that of waves at discontinuities in physical coefficients. These phenomena are well known [15], have been carefully analyzed [16] and numerically demonstrated [17]. 

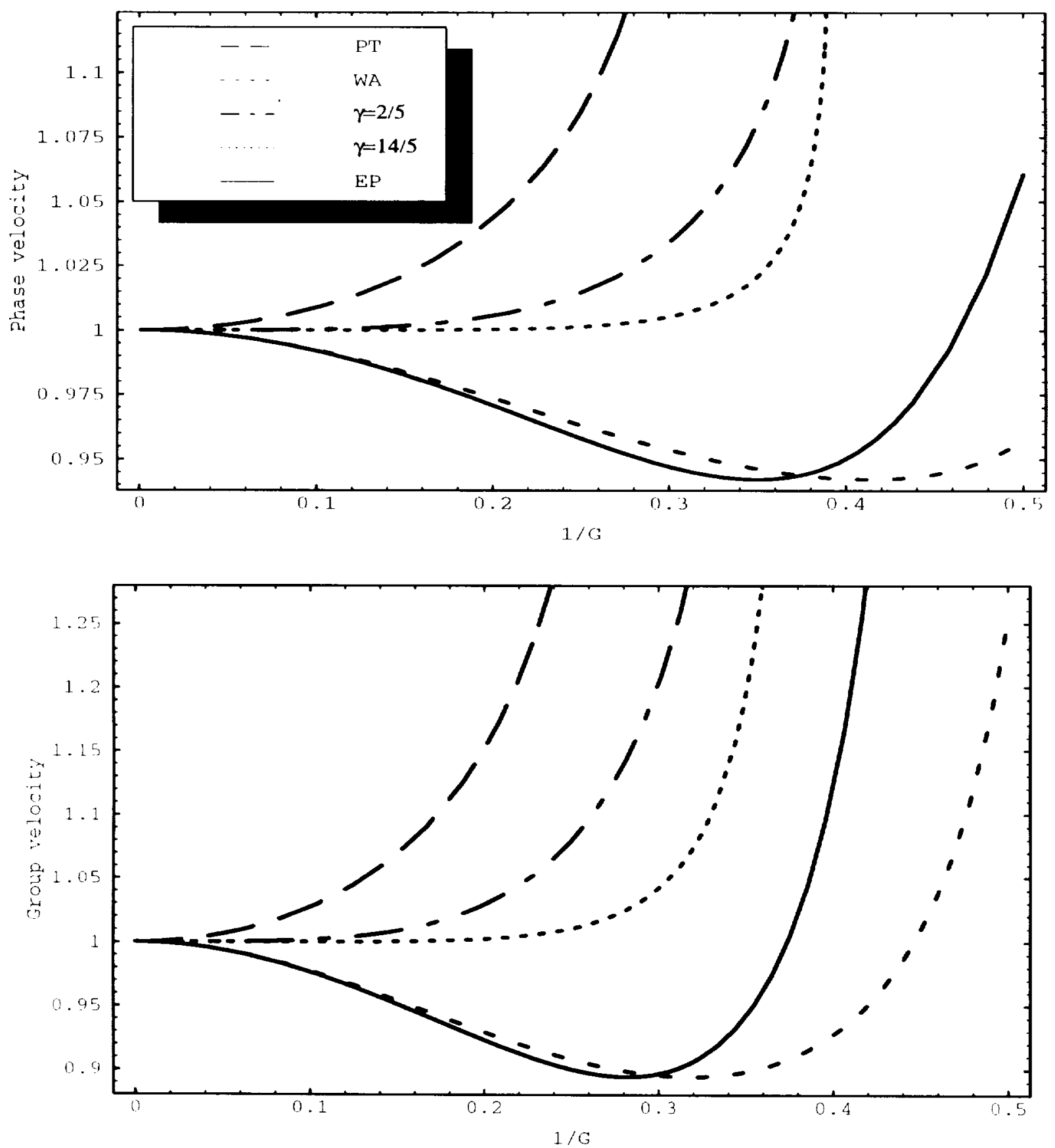

Figure 6: The ratio of numerical dispersion along grid lines and along cell diagonals for two-dimensional discrete formulations. 


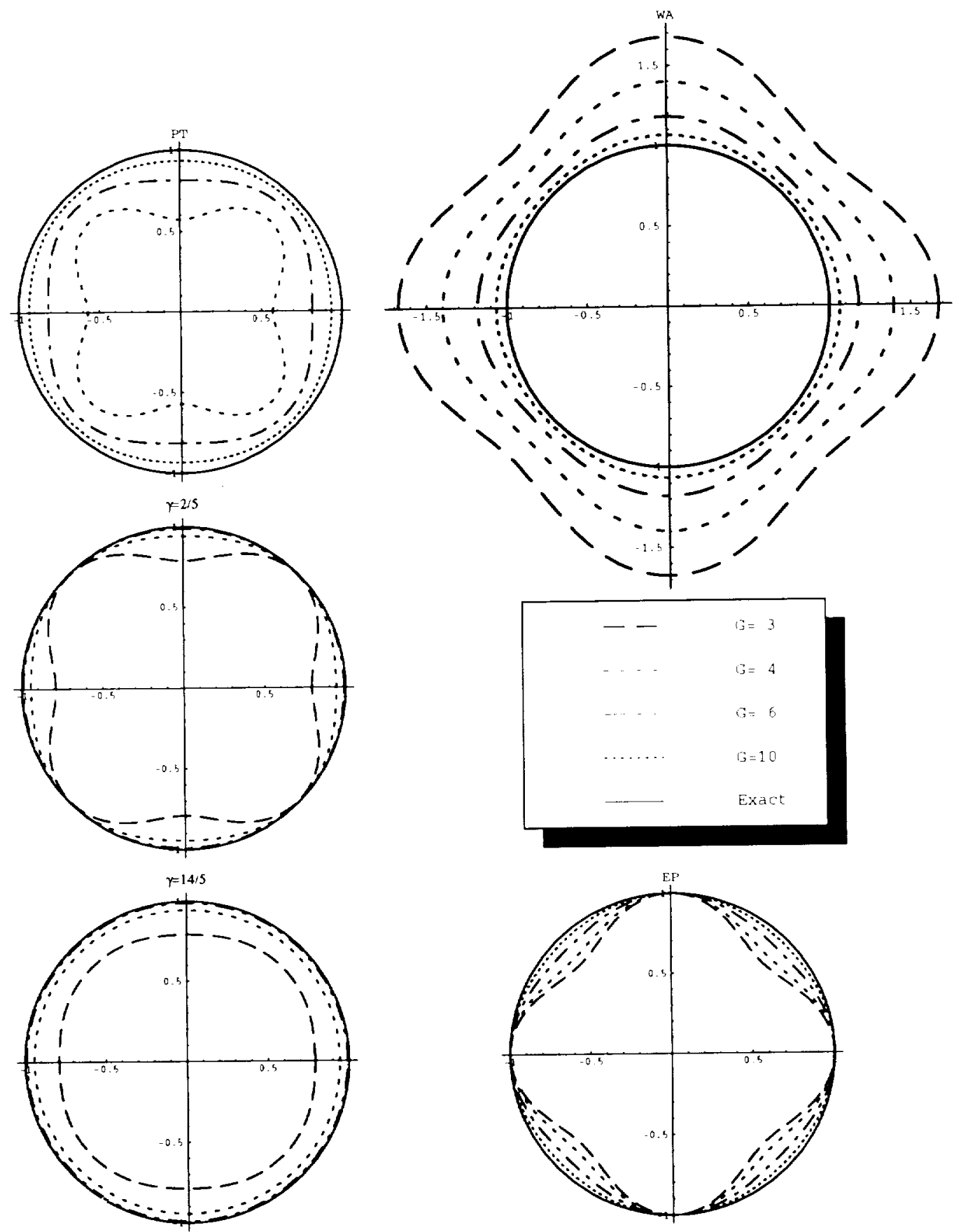

Figure 7: Polar plots of anisotropy in $\left(c_{p}^{h} / c_{0}\right)^{4}$ of two-dimensional discrete formulations. 
Consider a one-dimensional configuration discretized by a uniform grid of size $h^{-}$to the left of the origin and a uniform grid of size $h^{+}$to its right. Due to the transition in grid size at the origin, an incident plane wave of unit magnitude traveling in the positive direction with discrete values given in (33) for $j<0$ generates spurious transmission such that $\phi_{0} \neq 1$ and spurious reflection of magnitude $\phi_{0}-1$. The numerical solution is thus

$$
\phi_{j}= \begin{cases}\exp \left(i k^{h^{-}} h^{-}\right)^{j}+\left(\phi_{0}-1\right) / \exp \left(i k^{h^{-}} h^{-}\right)^{j}, & j<0 \\ \phi_{0} \exp \left(i k^{h^{+}} h^{+}\right)^{j}, & j \geq 0\end{cases}
$$

where the dispersion error is represented by the numerical wave numbers $k^{h^{ \pm}}=k^{h}\left(k h^{ \pm}\right)$, and the transmission error is represented by $\phi_{0}$. In particular

$$
\begin{aligned}
\phi_{1} & =\phi_{0} \exp \left(i k^{h^{+}} h^{+}\right) \\
\phi_{-1} & =\phi_{0} \exp \left(i k^{h^{-}} h^{-}\right)-2 i \sin \left(k^{h^{-}} h^{-}\right)
\end{aligned}
$$

PT The pointwise representation (4) of this solution at the origin yields

$$
\begin{aligned}
0= & \frac{2}{h^{+}+h^{-}}\left(\frac{\phi_{1}-\left(1-\left(k h^{+}\right)^{2} / 2\right) \phi_{0}}{h^{+}}+\frac{\phi_{-1}-\left(1-\left(k h^{-}\right)^{2} / 2\right) \phi_{0}}{h^{-}}\right) \\
= & \frac{2}{h^{+}+h^{-}}\left(\frac{\phi_{0} \exp \left(i k^{h^{+}} h^{+}\right)-\cos \left(k^{h^{+}} h^{+}\right) \phi_{0}}{h^{+}}+\right. \\
& \left.\frac{\phi_{0} \exp \left(i k^{h^{-}} h^{-}\right)-2 i \sin \left(k^{h^{-}} h^{-}\right)-\cos \left(k^{h^{-}} h^{-}\right) \phi_{0}}{h^{-}}\right) \\
= & \frac{2 i}{h^{+}+h^{-}}\left(\left(\frac{\sin \left(k^{h^{+}} h^{+}\right)}{h^{+}}+\frac{\sin \left(k^{h^{-}} h^{-}\right)}{h^{-}}\right) \phi_{0}-2 \frac{\sin \left(k^{h^{-}} h^{-}\right)}{h^{-}}\right)(59)
\end{aligned}
$$

where the second line was obtained by the dispersion relation for the pointwise representation (35). Thus

$$
\phi_{0}=\frac{2 \sqrt{1-\left(k h^{-}\right)^{2} / 4}}{\sqrt{1-\left(k h^{-}\right)^{2} / 4}+\sqrt{1-\left(k h^{+}\right)^{2} / 4}}
$$

which is valid in the range of resolution in which the pointwise formulation represents propagation (along grid lines). 
WA Similarly, the weighted-average representation (8) of the solution (56) at the origin leads to spurious transmission

$$
\phi_{0}=\frac{2 \sqrt{1-\left(k h^{-}\right)^{2} / 12}}{\sqrt{1-\left(k h^{-}\right)^{2} / 12}+\sqrt{1-\left(k h^{+}\right)^{2} / 12}}
$$

which is valid in the range of resolution in which the weighted-average formulation represents propagation (along grid lines).

HO Transmission for the higher-order representation with the parameter defined in $(15)$ is

$$
\phi_{0}=\frac{2 \frac{\sqrt{1-\left(k h^{-}\right)^{2} / 6}}{\left(1-\left(k h^{-}\right)^{2} / 12\right)}}{\frac{\sqrt{1-\left(k h^{-}\right)^{2} / 6}}{\left(1-\left(k h^{-}\right)^{2} / 12\right)}+\frac{\sqrt{1-\left(k h^{+}\right)^{2} / 6}}{\left(1-\left(k h^{+}\right)^{2} / 12\right)}}
$$

which is valid in the range of resolution in which the higher-order formulation represents propagation (along grid lines).

EP The case that is non-dispersive in one dimension (13) may be treated similarly. In this case the transmission is

$$
\phi_{0}=\frac{2 \frac{\sin \left(k h^{-}\right) / h^{-}}{2+\cos \left(k h^{-}\right)}}{\frac{\sin \left(k h^{-}\right) / h^{-}}{2+\cos \left(k h^{-}\right)}+\frac{\sin \left(k h^{+}\right) / h^{+}}{2+\cos \left(k h^{+}\right)}}
$$

which is valid in the range of resolution in which the higher-order formulation represents propagation (along grid lines).

Spurious transmission of the various formulations at different wave resolutions is plotted in Fig. 8. In general the sensitivity to transition in grid size is higher for coarser grids. The weighted-average representation is significantly superior to the pointwise scheme on non-uniform grids. The higher-order and exact-phase formulations offer further improvement. 

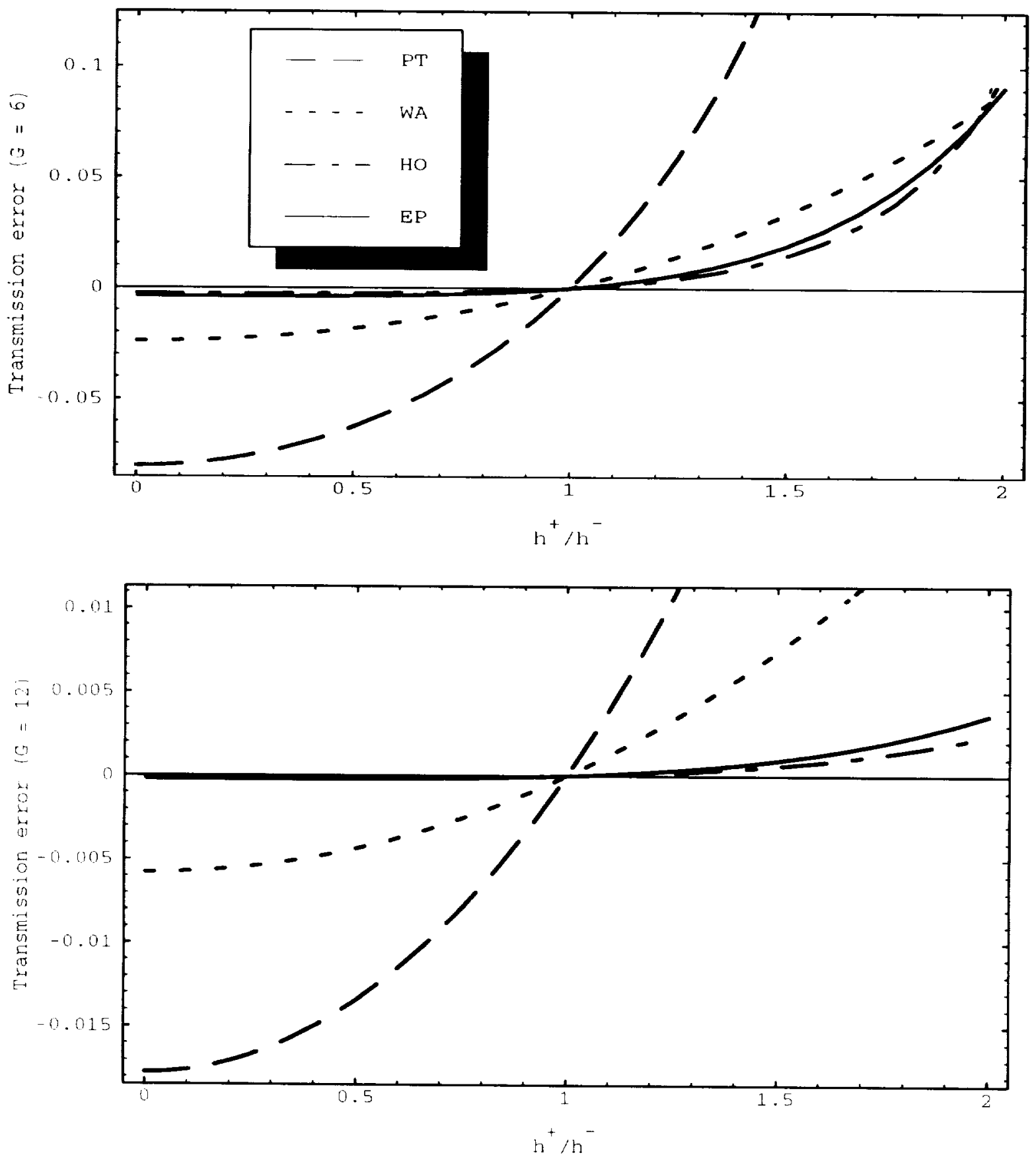

Figure 8: Transmission error $\left(\phi_{0}-1\right)$ at transition in grid size for 6 (top) and 12 grid points per wavelength to the left of the origin. 


\subsection{Interface of physical properties}

Discontinuities in physical properties give rise to wave reflection and transmission. The relative amplitudes of the reflected and transmitted waves depend on the ratio of wave numbers, which defines the character of the discontinuity. The numerical representation of these phenomena by finite element methods was studied in [18].

Consider a generalization of the previous configuration in which a discontinuity in material properties as well as a jump in grid size may occur at the origin, so that $k^{-}$is the wave number to the left of the origin and $k^{+}$-to its right. An incident plane wave of unit magnitude traveling in the positive direction $\exp \left(i k^{-} x\right)$ for $x<0$ generates reflected and transmitted waves, so that

$$
\phi= \begin{cases}\exp \left(i k^{-} x\right)+(\phi(0)-1) / \exp \left(i k^{+} x\right), & x<0 \\ \phi(0) \exp \left(i k^{+} x\right), & x \geq 0\end{cases}
$$

where

$$
\phi(0)=\frac{2 k^{-}}{k^{-}+k^{+}}
$$

The discrete solution is again (56) where the numerical wave numbers are $k^{h^{ \pm}}=k^{h}\left(k^{ \pm} h^{ \pm}\right)$.

PT The pointwise representation (5) of the solution at the origin yields

$$
\begin{aligned}
0 & =\frac{2}{h^{+}+h^{-}}\left(\frac{\phi_{1}-\left(1-\left(k^{+} h^{+}\right)^{2} / 2\right) \phi_{0}}{h^{+}}+\frac{\phi_{-1}-\left(1-\left(k^{-} h^{-}\right)^{2} / 2\right) \phi_{0}}{h^{-}}\right) \\
& =\frac{2 i}{h^{+}+h^{-}}\left(\left(\frac{\sin \left(k^{h^{+}} h^{+}\right)}{h^{+}}+\frac{\sin \left(k^{h^{-}} h^{-}\right)}{h^{-}}\right) \phi_{0}-2 \frac{\sin \left(k^{h^{-}} h^{-}\right)}{h^{-}}\right)
\end{aligned}
$$

where, again, the dispersion relation for the pointwise representation (35) is employed. Thus

$$
\phi_{0}=\frac{2 k^{-} \sqrt{1-\left(k^{-} h^{-}\right)^{2} / 4}}{k^{-} \sqrt{1-\left(k^{-} h^{-}\right)^{2} / 4}+k^{+} \sqrt{1-\left(k^{+} h^{+}\right)^{2} / 4}}
$$


WA Similarly, the weighted-average representation (9) of the solution (56) at the origin leads to

$$
\phi_{0}=\frac{2 k^{-} \sqrt{1-\left(k^{-} h^{-}\right)^{2} / 12}}{k^{-} \sqrt{1-\left(k^{-} h^{-}\right)^{2} / 12}+k^{+} \sqrt{1-\left(k^{+} h^{+}\right)^{2} / 12}}
$$

HO Transmission for the higher-order representation with the parameter defined in (15) is

$$
\phi_{0}=\frac{2 k^{-} \frac{\sqrt{1-\left(k^{-} h^{-}\right)^{2} / 6}}{\left(1-\left(k^{-} h^{-}\right)^{2} / 12\right)}}{k^{-} \frac{\sqrt{1-\left(k^{-} h^{-}\right)^{2} / 6}}{\left(1-\left(k^{-} h^{-}\right)^{2} / 12\right)}+k^{+} \frac{\sqrt{1-\left(k^{+} h^{+}\right)^{2} / 6}}{\left(1-\left(k^{+} h^{+}\right)^{2} / 12\right)}}
$$

EP The case that is non-dispersive in one dimension (13) may be treated similarly. In this case the transmission is

$$
\phi_{0}=\frac{2 k^{-} \frac{\sin \left(k h^{-}\right) /\left(k^{-} h^{-}\right)}{2+\cos \left(k h^{-}\right)}}{k^{-} \frac{\sin \left(k h^{-}\right) /\left(k^{-} h^{-}\right)}{2+\cos \left(k h^{-}\right)}+k^{+} \frac{\sin \left(k h^{+}\right) /\left(k^{+} h^{+}\right)}{2+\cos \left(k h^{+}\right)}}
$$

Physical transmission depends on the ratio of the wave numbers. Numerical solutions depend on this parameter and on the ratio of resolutions. To find out which of the two parameters significantly effects the numerical error in transmission consider the transmission error as a function of ratio of resolutions for 6 grid points per wavelength to the left of the origin. This is plotted for a ratio of the wave numbers equal to unity in Fig. 8 (top). Increasing the ratio by one order of magnitude and by two yields the behavior shown in Fig. 9. The difference between these plots is not significant indicating that the error depends primarily on the ratio of resolutions. All the representations have the property that $\phi_{0}=\phi(0)$ if $k^{+} h^{+}=k^{-} h^{-}$. Again, superior performance of the weighted-average representation and its enhancements is evident. 

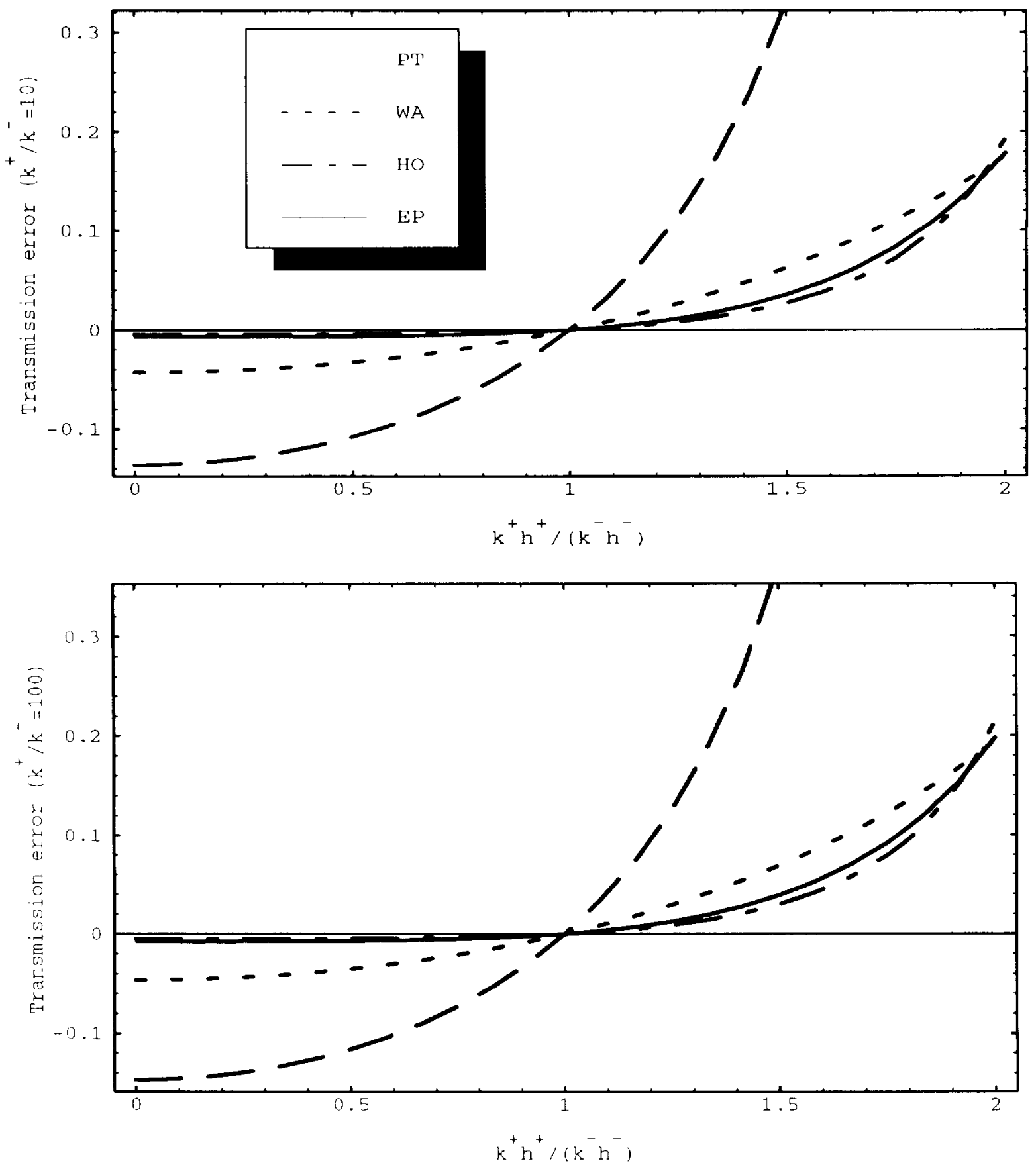

Figure 9: Transmission error $\left(\phi_{0}-\phi(0)\right) / \phi(0)$ at wave number ratios of 10 (top) and 100 for 6 grid points per wavelength to the left of the origin. 


\section{Local Truncation Error Analysis}

The local truncation error is the residual left by substituting the exact solution in the discrete representation. In the following, sufficient differentiability is assumed for all functions involved.

\subsection{Uniform grids}

Consider the one-dimensional constant-coefficient case on a uniform grid. For the pointwise representation (3)

$$
\begin{aligned}
\tau & =\frac{\phi\left(x_{j+1}\right)-2 \phi\left(x_{j}\right)+\phi\left(x_{j-1}\right)}{h^{2}}+k^{2} \phi\left(x_{j}\right)+f\left(x_{j}\right) \\
& =\phi^{\prime \prime}\left(x_{j}\right)+\frac{h^{2}}{12} \phi^{i v}\left(x_{j}\right)+\frac{h^{4}}{180}\left(\phi^{v i}\left(x^{+}\right)+\phi^{v i}\left(x^{-}\right)\right)+k^{2} \phi\left(x_{j}\right)+f\left(x_{j}\right) \\
& =\frac{h^{2}}{12} \phi^{i v}\left(x_{j}\right)+O\left(h^{4}\right)
\end{aligned}
$$

where primes and superior Roman numerals indicate differentiation by the argument. The second line is obtained by Taylor's formula, where $x_{j-1} \geq$ $x^{-} \geq x_{j}$ and $x_{j} \geq x^{+} \geq x_{j+1}$, and the third line, which follows from the fact that $\phi$ satisfies the Helmholt $z$ equation, indicates consistency. The pointwise scheme is thus second-order accurate.

The weighted-average representation (6) is similar on uniform grids

$$
\begin{aligned}
\tau= & \frac{\phi\left(x_{j+1}\right)-2 \phi\left(x_{j}\right)+\phi\left(x_{j-1}\right)}{h^{2}}+k^{2} \frac{\phi\left(x_{j+1}\right)+4 \phi\left(x_{j}\right)+\phi\left(x_{j-1}\right)}{6}+ \\
= & \left(1+\frac{f\left(x_{j+1}\right)+4 f\left(x_{j}\right)+f\left(x_{j-1}\right)}{6}\right) \frac{\phi\left(x_{j+1}\right)-2 \phi\left(x_{j}\right)+\phi\left(x_{j-1}\right)}{h^{2}}+k^{2} \phi\left(x_{j}\right)+ \\
& f\left(x_{j}\right)+\frac{f\left(x_{j+1}\right)-2 f\left(x_{j}\right)+f\left(x_{j-1}\right)}{6} \\
= & -\frac{h^{2}}{12} \phi^{i v}\left(x_{j}\right)+O\left(h^{4}\right)
\end{aligned}
$$

The weighted-average scheme is also second-order accurate. This order of accuracy is retained in the case of variable coefficients (7). 
For the improved representations (14)

$$
\begin{aligned}
\tau= & \frac{\phi\left(x_{j+1}\right)-2 \phi\left(x_{j}\right)+\phi\left(x_{j-1}\right)}{\beta h^{2}}+k^{2} \frac{\phi\left(x_{j+1}\right)+4 \phi\left(x_{j}\right)+\phi\left(x_{j-1}\right)}{6}+ \\
\frac{f\left(x_{j+1 / 2}\right)+f\left(x_{j}\right)+f\left(x_{j-1 / 2}\right)}{3} & \left(1+\frac{\beta(k h)^{2}}{6}\right) \frac{\phi\left(x_{j+1}\right)-2 \phi\left(x_{j}\right)+\phi\left(x_{j-1}\right)}{\beta h^{2}}+k^{2} \phi\left(x_{j}\right)+ \\
& f\left(x_{j}\right)+\frac{f\left(x_{j+1 / 2}\right)-2 f\left(x_{j}\right)+f\left(x_{j-1 / 2}\right)}{3}
\end{aligned}
$$

Employing the definition of $\beta$ that leads to the high-order representation (15) yields

$$
\tau=\frac{h^{4}}{240}\left(k^{2} \phi^{i v}\left(x_{j}\right)-f^{i v}\left(x_{j}\right) / 4\right)+O\left(h^{6}\right)
$$

justifying its name as a higher-order scheme. Note that if the source terms were not represented appropriately there would be second-order terms in the truncation error. The scheme that is dispersion-free in one dimension (13) has a truncation error

$$
\tau=\frac{h^{4}}{240}\left(k^{2}\left(\phi^{i v}\left(x_{j}\right)+k^{2} \phi^{\prime \prime}\left(x_{j}\right)\right)-f^{i v}\left(x_{j}\right) / 4\right)+O\left(h^{6}\right)
$$

If the fourth derivative of the source vanishes the method becomes six-order accurate. Furthermore, the truncation error is zero when all the derivatives of the source from fourth order and higher vanish.

\subsection{Non-uniform grids}

In analyzing method performance on non-uniform grids a change of variables from physical space to computational space is often considered, so that the grid is uniform in the latter [19]. The order of accuracy of some methods on non-uniform grids may drop in physical space. Nevertheless, in computational space it remains unchanged from the order in the uniform case. To a certain extent grid stretching should reflect variation of the solution. In this case accuracy in computational space is representative of the situation. In

practice, however, grid variation is determined by geometric considerations 
as well. The following results are thus presented in physical space, which describes the more general case.

For the pointwise representation (4)

$$
\begin{aligned}
\tau= & \left(\frac{\phi\left(x_{j+1}\right)-\phi\left(x_{j}\right)}{h^{+}}-\frac{\phi\left(x_{j}\right)-\phi\left(x_{j-1}\right)}{h^{-}}\right) / \frac{h^{+}+h^{-}}{2}+k^{2} \phi\left(x_{j}\right)+f\left(x_{j}\right) \\
= & \phi^{\prime \prime}\left(x_{j}\right)+\frac{h^{+}-h^{-}}{3} \phi^{\prime \prime \prime}\left(x_{j}\right)+\frac{\left(h^{+}\right)^{2}-h^{+} h^{-}+\left(h^{-}\right)^{2}}{12} \phi^{i v}\left(x_{j}\right)+ \\
& \quad \frac{1}{60\left(h^{+}+h^{-}\right)}\left(\left(h^{+}\right)^{4} \phi^{v}\left(x^{+}\right)-\left(h^{-}\right)^{4} \phi^{v}\left(x^{-}\right)\right)+k^{2} \phi\left(x_{j}\right)+f\left(x_{j}\right) \\
= & \frac{h^{+}-h^{-}}{3} \phi^{\prime \prime \prime}\left(x_{j}\right)+\frac{\left(h^{+}\right)^{2}-h^{+} h^{-}+\left(h^{-}\right)^{2}}{12} \phi^{i v}\left(x_{j}\right)+O\left(h^{3}\right)
\end{aligned}
$$

The pointwise scheme is indeed second-order accurate on uniform grids, but may drop to first order in the non-uniform case.

Whether the scheme actually drops to first order or not depends on the degree of grid stretching. If

$$
h^{+}-h^{-}=O\left(h^{p+1}\right), \quad p>0
$$

the stretching is called algebraic [19]. With algebraic stretching the pointwise scheme retains second-order accuracy. Otherwise the accuracy drops to first order.

In contrast, for the weighted-average representation (8)

$$
\begin{aligned}
\tau= & \left(\frac{\phi\left(x_{j+1}\right)-\phi\left(x_{j}\right)}{h^{+}}-\frac{\phi\left(x_{j}\right)-\phi\left(x_{j-1}\right)}{h^{-}}\right) / \frac{h^{+}+h^{-}}{2}+ \\
& \frac{k^{2}}{3}\left(\frac{h^{+}}{h^{+}+h^{-}}\left(\phi\left(x_{j+1}\right)+2 \phi\left(x_{j}\right)\right)+\frac{h^{-}}{h^{+}+h^{-}}\left(2 \phi\left(x_{j}\right)+\phi\left(x_{j-1}\right)\right)\right)+ \\
& \frac{1}{3}\left(\frac{h^{+}}{h^{+}+h^{-}}\left(f\left(x_{j+1}\right)+2 f\left(x_{j}\right)\right)+\frac{h^{-}}{h^{+}+h^{-}}\left(2 f\left(x_{j}\right)+f\left(x_{j-1}\right)\right)\right) \\
= & \frac{2}{h^{+}+h^{-}}\left(\left(1+\frac{\left(k h^{+}\right)^{2}}{6}\right) \frac{\phi\left(x_{j+1}\right)-\phi\left(x_{j}\right)}{h^{+}}+\right. \\
& \left.\left(1+\frac{\left(k h^{-}\right)^{2}}{6}\right) \frac{\phi\left(x_{j-1}\right)-\phi\left(x_{j}\right)}{h^{-}}\right)+k^{2} \phi\left(x_{j}\right)+ \\
& f\left(x_{j}\right)+\frac{1}{3\left(h^{+}+h^{-}\right)}\left(h^{+}\left(f\left(x_{j+1}\right)-f\left(x_{j}\right)\right)+h^{-}\left(f\left(x_{j}\right)-f\left(x_{j-1}\right)\right)\right)
\end{aligned}
$$




$$
=-\frac{\left(h^{+}\right)^{2}-h^{+} h^{-}+\left(h^{-}\right)^{2}}{12} \phi^{i v}\left(x_{j}\right)+O\left(h^{3}\right)
$$

first-order terms cancel out even on non-uniform grids so that second-order accuracy is retained in any case. Again, these results apply to the case of variable coefficients as well.

For the improved representations

$$
\begin{gathered}
\tau=\left(\frac{\phi\left(x_{j+1}\right)-\phi\left(x_{j}\right)}{h^{+}}-\frac{\phi\left(x_{j}\right)-\phi\left(x_{j-1}\right)}{h^{-}}\right) / \frac{\beta^{+} h^{+}+\beta^{-} h^{-}}{2}+ \\
\quad \frac{k^{2}}{3}\left(\frac{\beta^{+} h^{+}}{\beta^{+} h^{+}+\beta^{-} h^{-}}\left(\phi\left(x_{j+1}\right)+2 \phi\left(x_{j}\right)\right)\right. \\
\left.\quad+\frac{\beta^{-} h^{-}}{\beta^{+} h^{+}+\beta^{-} h^{-}}\left(2 \phi\left(x_{j}\right)+\phi\left(x_{j-1}\right)\right)\right)+ \\
\quad \frac{\beta^{+} h^{+}}{3}\left(2 f\left(x_{j+1 / 2}\right)+f\left(x_{j}\right)\right) \\
\left.\quad+\frac{\beta^{-} h^{-}}{\beta^{+} h^{+}+\beta^{-} h^{-}}\left(f\left(x_{j}\right)+2 f\left(x_{j-1 / 2}\right)\right)\right) \\
\frac{2}{\beta^{+} h^{+}+\beta^{-} h^{-}}\left(\left(1+\frac{k^{2} \beta^{+} h^{+} h^{+}}{6}\right) \frac{\phi\left(x_{j+1}\right)-\phi\left(x_{j}\right)}{h^{+}}+\right. \\
\left.\left(1+\frac{k^{2} \beta^{-} h^{-} h^{-}}{6}\right) \frac{\phi\left(x_{j-1}\right)-\phi\left(x_{j}\right)}{h^{-}}\right)+k^{2} \phi\left(x_{j}\right)+ \\
f\left(x_{j}\right)+\frac{2}{3\left(\beta^{+} h^{+}+\beta^{-} h^{-}\right)}\left(\beta^{+} h^{+}\left(f\left(x_{j+1 / 2}\right)-f\left(x_{j}\right)\right)\right. \\
\left.+\beta^{-} h^{-}\left(f\left(x_{j}\right)-f\left(x_{j-1 / 2}\right)\right)\right)
\end{gathered}
$$

For the definition of $\beta$ that leads to the high-order representation (15)

$$
\begin{aligned}
& \tau=\frac{\left(h^{+}-h^{-}\right)\left(\left(h^{+}\right)^{2}+\left(h^{-}\right)^{2}\right)}{90}\left(k^{2} \phi^{\prime \prime \prime}\left(x_{j}\right)-f^{\prime \prime}\left(x_{j}\right) / 4\right)+ \\
& \frac{\left(h^{+}\right)^{4}-\left(h^{+}\right)^{3} h^{-}+\left(h^{+}\right)^{2}\left(h^{-}\right)^{2}-h^{+}\left(h^{-}\right)^{3}+\left(h^{-}\right)^{4}}{240}\left(k^{2} \phi^{i v}\left(x_{j}\right)-f^{i v}\left(x_{j}\right) / 4\right)+ \\
& O\left(h^{5}\right)
\end{aligned}
$$

the truncation error is third-order accurate on non-uniform grids (and, of course, fourth order in the uniform case). 


\section{Conclusions}

In this work finite difference methods for solving problems of time-harmonic acoustics are developed and analyzed. The well-known pointwise representation, eqs. (3) and (19) in one and two dimensions, respectively, is secondorder accurate on uniform grids. However, accuracy may drop to first order in the non-uniform case (4) unless sufficiently smooth grid stretching (77) is employed. In multi-dimensional configurations the representation actually improves the less aligned the propagation directions are with respect to the grid.

A weighted-average representation, eqs. (6) and (21) in one and two dimensions, respectively, has the same asymptotic behavior on uniform grids, but is less sensitive to low wave resolution and, more importantly, to direction of propagation and transition in wave resolution (including material interfaces). Performance in multi-dimensional configurations again improves for propagation directions that are not aligned with the grid. In general, anisotropy in numerical representation is reduced with increased wave resolution. At lower resolution the weighted-average representation (21) is much less anisotropic than the standard pointwise representation (19). Secondorder accuracy is retained on any non-uniform grid (8) at virtually no increase in computational cost. These results hold for variable coefficients as well.

Superior performance is attained by basing the schemes on a generalized definition of the derivative (10) which incorporates a resolution-dependent

parameter. Improved schemes with higher-order accuracy are designed by appropriate definition of the parameter (15), reducing spurious dispersion and reflection. Defining the parameter for schemes which are, in some cases, dispersion-free (13) leads to the same asymptotic behavior with improved coarse grid accuracy. Source terms must be represented accordingly (14) so as not to degrade the higher-order accuracy. These methods are, in general, fourth-order accurate on uniform grids and third order in the non-uniform case. The performance of these schemes in multi-dimensional configurations is superior for any direction of propagation. Their performance improves as propagation directions become aligned with the grid. In principle, grids should thus be aligned with directions of propagation to the extent possible, further enhancing the performance of these methods.

Schemes that exhibit higher-order behavior on uniform grids in all di- 
rections in two-dimensional configurations are derived on the basis of Padé approximation and its generalization (22). The dispersion of these methods (as well as their spurious reflection and transmission) along grid lines is identical to that of the higher-order method based on the generalized definition of the derivative. Dispersion along grid diagonals is minimized by employing $\gamma=2 / 5$ which leads to (27). These methods are by far less anisotropic than all other schemes. The value of $\gamma=14 / 5$ leads to the stencil with the lowest degree of anisotropy (28).

In general, wave resolution $(k h)$ should be kept as even as possible throughout the grid to minimize spurious reflection and transmission. Sensitivity to these phenomena is greater on relatively coarse grids.

\section{Acknowledgement}

The authors wish to thank Saul Abarbanel, Najib Abboud, Alvin Bayliss, Greg Hulbert, Jim Stewart and Jay Webb for helpful comments and discussions.

\section{References}

[1] R.E. Lynch and J.R. Rice, "A higher-order difference method for differential equations," Math. Comput. 34(150) (1980) 333-372.

[2] R.E. Lynch and J.R. Rice, "The HODIE method and its performance for solving elliptic partial differential equations," in: C. de Boor and G.H. Golub, eds., Recent Advances in Numerical Analysis (Academic Press, New York, 1978) 143-175.

[3] R.E. Lynch, " $O\left(h^{4}\right)$ and $O\left(h^{6}\right)$ finite difference approximations to the Helmholtz equation in $n$ dimensions," in: R. Vichnevetsky and R.S. Stepleman, eds., Advances in Computer Methods for Partial Differential Equations, Vol. V (IMACS, New Brunswick, NJ, 1984) 199-202.

[4] S.K. Lele, "Compact finite difference schemes with spectral-like resolution," J. Comput. Phys. 103 (1992) 16-42. 
[5] C.K.W. Tam and J.C. Webb, "Dispersion-relation-preserving finite difference schemes for computational acoustics," J. Comput. Phys. 107 (1993) 262-281.

[6] R.E. Mickens, "Construction of stable explicit finite-difference schemes for Schrödinger type differential equations," Comput. Acou. 1 (1990) $11-16$.

[7] R.E. Mickens and J.N. Shoosmith "A discrete model of a modified Burgers' partial differential equation," Journal of Sosnd and Vibration 142(3) (1990) 536-539.

[8] I. Harari and T.J.R. Hughes, "Galerkin/least-squares finite element methods for the reduced wave equation with non-reflecting boundary conditions in unbounded domains," Comput. Methods in Appl. Mech. Engrg. 98 (1992) 411-454.

[9] A. Bayliss, C.I. Goldstein and E. Turkel, "On accuracy conditions for the numerical computation of waves," J. Comput. Phys. 59 (1985) 376-404.

[10] P. Wesseling, An Introduction to Multigrid Methods (Wiley, Chichester, 1992).

[11] T.J.R. Hughes, The Finite Element Method: Linear Static and Dynamic Finite Element Analysis (Prentice-Hall, Englewood Cliffs, New Jersey, 1987).

[12] L. Collatz, The Numerical Treatment of Differential Equations, third ed. (Springer-Verlag, Berlin, 1960).

[13] G.L. Goudreau and R.L. Taylor, "Evaluation of numerical integration methods in elastodynamics," Comput. Methods in Appl. Mech. Engrg. 2 (1972) 69-97.

[14] L.L. Thompson and P.M. Pinsky, "A Galerkin least squares finite element method of the two-dimensional Helmholtz equation," Internat. $J$. Numer. Methods Engrg to appear.

[15] T.B. Belytschko, "Transient analysis," in: Structural Mechanics Computer Programs (Univ. Press of Virginia, Charlottesville, VA, 1974) 255276. 
[16] Z.P. Bažant, "Spurious reflection of elastic waves in nonuniform finite element grids," Comput. Methods in Appl. Mech. Engrg. 16 (1978) 91100.

[17] I. Fried, "Accuracy of string element mass matrix," Comput. Methods in Appl. Mech. Engrg. 20 (1979) 317-321.

[18] L. Jiang and R.J. Roberts, "Spurious wave reflections at an interface of different physical properties in finite-element wave solutions," Commun. in Appl. Numer. Methods 7 (1991) 565-602.

[19] E. Turkel, "Accuracy of schemes with nonuniform meshes for compressible fluid flows," Appl. Numer. Math. 2 (1986) 529-550. 
Public reporting burden for this collection of information is estimaled to average 1 hour per response, including the time for reviewing instructions, searching existing data sources. gathering and maintaining the data needed, and completing and reviewing the collection of information. Send comments regarding this burden estimate or any other aspect of this theadpuarters Services, Direciorate for Information Operations and Keporis $12, \mathrm{~h}$ Jefferson Davis Highway, Suite 1204. Arlington. VA 22202 430? and to the Office of Management and Rudgel, Paperwork Reduction Project (0/04.0148). Washington, D) 2050?

\begin{tabular}{|l|l|l} 
1. AGENCY USE ONLY(leave blank) & $\begin{array}{l}\text { 2. REPORT DATE } \\
\text { March } 1994\end{array}$ & $\begin{array}{l}\text { 3. REPORT TYPE AND DATES COVERED } \\
\text { (iontractor Report }\end{array}$
\end{tabular}

4. TITLE AND SUBTITLE

AOCLRATF FINITE DIFFERENCE WEJHOIST FOR

TIME-HARMONIC WAVE PROPACIATION

\section{FUNDING NUMBERS}

(: VAsil-1!4811

W[ $5,0,5-9(1)-72-0)]$

\section{AUTHOR(S)}

Isaac Harari

Eli Turkul

7. PERFORMING ORGANIZATION NAME(S) AND ADDRESS(ES)

lustitute for computer Applications in seinence

and Fongineering

Mail Sitop 1326, NASA Langley Research Genter

Contractor Report

Hamptou, VA 2:3681-00011

9. SPONSORING/MONITORING AGENCY NAME(S) AND ADDRESS(ES)

National Acromantics and Space Administration

Jangley Research ('enter

Jampton, VA $23681-0001$

8. PERFORMING ORGANIZATION REPORT NUMBER

lo'Atri Report No. 94-13

10. SPONSORING/MONITORING AGENCY REPORT NUMBER

NASA (:R-IMAR8T

ICASER Report No. 41-13

\section{SUPPLEMENTARY NOTES}

langley Technical Monitur: Michael F. Card

Final Report

Submitted to Journal of Computational Physics

\begin{tabular}{l|l|l} 
12a. DISTRIBUTION/AVAILABILITY STATEMENT & 12b. DISTRIBUTION CODE
\end{tabular}

Inclassified tinlimited

Subject category 64

13. ABSTRACT (Maximum 200 words)

Finite difference methods for solving problems of time-harmonic aconstics are developed and analyzed. Alultidimensional inhonogeneous problems with variable, possibly discontinuons, coefficients are considered. accumbing for the effects of employing non-mniformeriels. A weighted-average representation is less sensitive to transition in wave resolution (due to variable wave numbers or non-uniform grids) than the standard pointwise representation. Further enhancement in method performance is obtained hy basing the stencis on generalizations of Pade approximation, or generalized definitions of the derivative, reducing spurións dispersion, anisotropy and reflextion, and by improving the representation of source terms. The resulting schemes have fourth-order accurate local truncation error on mniform grids and third order in the non-moform case. Cindelines for discretization pertaining to grid orientation and resolution are presented.

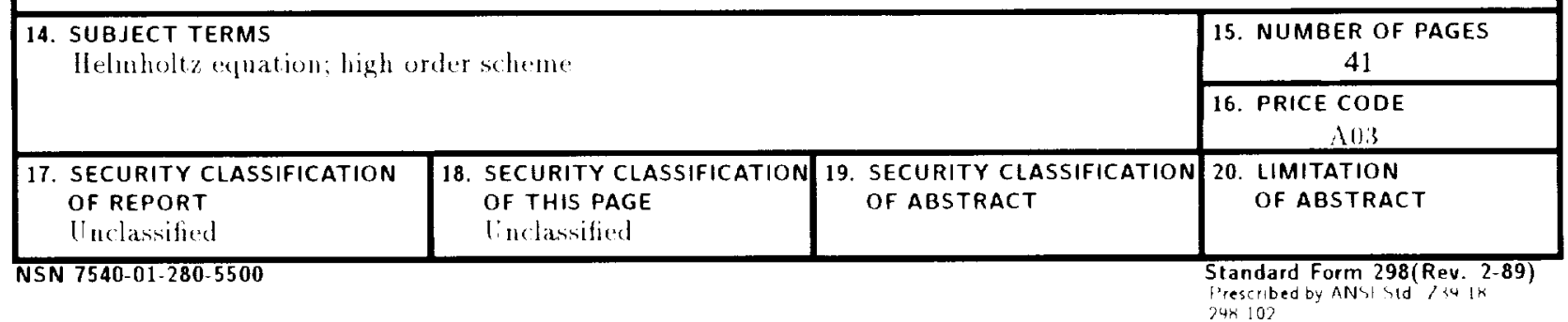


